\title{
ARTICLE
}

Check for

Check for
updates

Cite as

Nano-Micro Lett.

(2022) 14:24

Received: 28 September 2021

Accepted: 16 November 2021

Published online: 9 December 2021

(c) The Author(s) 2021

\section{Fabrication of High-Density Out-of-Plane Microneedle Arrays with Various Heights and Diverse Cross-Sectional Shapes}

\author{
Hyeonhee Roh ${ }^{1,2} \mathbb{D}$, Young Jun Yoon $^{1}$, Jin Soo Park ${ }^{1,2} \mathbb{D}^{-}$, Dong-Hyun Kang ${ }^{3}$, \\ Seung Min Kwak ${ }^{3}$, Byung Chul Lee ${ }^{1} \mathbb{D}$, Maesoon $\operatorname{Im}^{1,4} \mathbb{C}^{\square}$
}

\section{HIGHLIGHTS}

- High-density out-of-plane microneedle arrays were fabricated with a single photolithography and two deep reactive ion etching (DRIE) steps in anisotropic and isotropic modes, respectively.

- Microneedles in various heights were monolithically created by the identical DRIE processes and scanning electron microscopy images showed extremely sharp sub-micron ( 145-nm-wide) tip.

- Diverse cross-sectional shapes of microneedles were implemented by altering photomask patterns.

\begin{abstract}
Out-of-plane microneedle structures are widely used in various applications such as transcutaneous drug delivery and neural signal recording for brain machine interface. This work presents a novel but simple method to fabricate high-density silicon (Si) microneedle arrays with various heights and diverse cross-sectional shapes depending on photomask pattern designs. The proposed fabrication method is composed of a single photolithography and two subsequent deep reactive ion etching (DRIE) steps. First, a photoresist layer was patterned on a Si substrate to define areas to be etched, which will eventually determine the final location and shape of each individual microneedle. Then, the 1st DRIE step

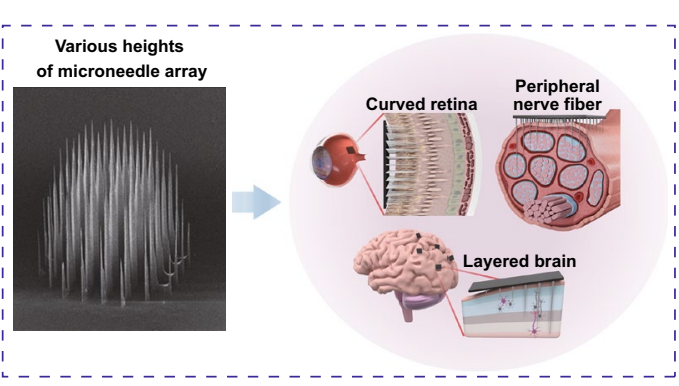
created deep trenches with a highly anisotropic etching of the Si substrate. Subsequently, the photoresist was removed for more isotropic etching; the 2nd DRIE isolated and sharpened microneedles from the predefined trench structures. Depending on diverse photomask designs, the 2nd DRIE formed arrays of microneedles that have various height distributions, as well as diverse cross-sectional shapes across the substrate. With these simple steps, high-aspect ratio microneedles were created in the high density of up to 625 microneedles $\mathrm{mm}^{-2}$ on a Si wafer. Insertion tests showed a small force as low as $\sim 172 \mu \mathrm{N} /$ microneedle is required for microneedle arrays to penetrate the dura mater of a mouse brain. To demonstrate a feasibility of drug delivery application, we also implemented silk microneedle arrays using molding processes. The fabrication method of the present study is expected to be broadly applicable to create microneedle structures for drug delivery, neuroprosthetic devices, and so on.
\end{abstract}

KEYWORDS Microneedle; Various heights; Cross-sectional shapes; Isotropic etch; Deep reactive ion etching

Hyeonhee Roh, Young Jun Yoon, Jin Soo Park, and Dong-Hyun Kang contributed equally to this work.

$\triangle$ Maesoon Im, maesoon.im@kist.re.kr

1 Brain Science Institute, Korea Institute of Science and Technology (KIST), Seoul 02792, South Korea

2 Division of Electrical Engineering, College of Engineering, Korea University, Seoul 02841, South Korea

3 Micro/Nano Fabrication Center, KIST, Seoul 02792, South Korea

4 Division of Bio-Medical Science \& Technology, KIST School, University of Science \& Technology (UST), Seoul 02792, South Korea 


\section{Introduction}

High-aspect ratio microneedle structures have long been studied for various applications. For example, it has been well known that micro-sized needle arrays can deliver drugs without pain that would be caused by the insertion of macro-sized syringe needles [1]. It is because microneedles can reach down to the epidermis layer at a limited penetration depth without irritating dermis layers associated with pain and tissue damage $[2,3]$. Thus, microneedles in various shapes have been used for transcutaneous delivery of diverse drugs [4-8] even including recombinant COVID-19 vaccines $[9,10]$. Recent publications have also shown that microneedles can be applied to blood vessels [11], vesicles [12], corneas [13], heart [14], and plants [15, 16], demonstrating a possibility of a much broader array of applications. Furthermore, microneedle structures have been widely used in numerous researches of modern neuroscience and neural engineering [17]. Particularly, neural probes in a shape of out-of-plane microneedle arrays are critical for recording various neural signals ranging from single unit activities [18, 19] to electroencephalogram (EEG) signals [20]. In addition to the electrical recording of neural activities, microneedle arrays can be used for the electrical stimulation of nerve cells in the central/peripheral nervous systems [21-23]. These examples suggest that there are and will be increasing demands on microneedle arrays as neural interface devices.

In both applications (i.e., neural interface and drug delivery), the following three aspects of microneedles can be considered. First, high-density microneedle arrays would be preferred for more effective performance. For instance, high-density microneedle electrodes (i.e., highchannel counts) would better discern complex neural signals from densely populated neurons or nerve fibers, enabling more sophisticated functions of brain machine interfaces (BMIs) due to increased selectivity [17, 21, 24]. Also, there have been numerous literature works reporting the high density of microneedles in diverse fabrication methods to enhance the drug delivery efficiency [3, 25-27]. Second, microneedles in various heights (or lengths) would enable more comprehensive interrogation of neurons located at different depths of the complex three-dimensional nervous systems such as laminated cortex areas [28] and peripheral nerves [22, 29]. In case of electric stimulation as well, convoluted shapes of microelectrode arrays are required for specific applications such as nerve fiber or retinal stimulations [30, 31]. This is because the central/peripheral nervous system consists of three-dimensional structures with several layers which have distinctive functions [22, 32, 33]; microneedles in various heights can stimulate those different layers of a nervous system individually or at once. Third, the crosssectional shape of microneedles would be also of importance for the effective insertion to various target tissues such as brain or skin. Particularly, difficulties in device insertion of high-density microneedles often limit appropriate performances: for example, a pneumatic inserter was needed to implant Utah arrays to the brain tissue [34, 35] because numerous microneedles as a whole require big penetration forces. It has been known that the necessary insertion force can be substantially reduced with a smaller diameter [36] and/or increased sharpness of microstructures [37-39]. Moreover, a sharp tip of neuroprosthetic devices is preferred to minimize insertion damage of neural tissues [40-43], as well as to reduce foreignbody response $[44,45]$. In certain applications, specific cross-sectional shapes would be preferred for reducing the puncture resistance, such as polygonal and star-shaped microneedles [46].

Conventional fabrication methods are not appropriate for monolithic fabrication of high-density out-of-plane microneedle arrays in varying heights and diverse crosssectional shapes (i.e., satisfying the abovementioned three features at once). For example, the Utah arrays for neural applications are manufactured by a series of steps including dicing of silicon $(\mathrm{Si})$ wafers, and wet etching of resulting Si pillars [30, 47]. Since dicing kerf lines $(\sim 100 \mu \mathrm{m})$ determines both dimension and density of microneedle arrays, the high-density array with small feature size has long been challenging to be obtained. In the Utah arrays, 25 electrodes $\mathrm{mm}^{-2}$ seems to be the record to date [23]. For drug delivery applications as well, microneedles have been fabricated by complex etching processes $[1,48]$ or other methods such as the drawing lithography [49-51]. These existing fabrication technologies are too complex or do not allow various cross-sectional shapes of final microneedle structures which may be beneficial in other applications.

In the present study, we report a novel but simple fabrication method for high-density out-of-plane microneedle arrays that can have various heights in arbitrary distribution 
and diverse cross-sectional shapes depending on photomask pattern designs. The proposed method consists of one single photolithography and two subsequent deep reactive ion etching (DRIE) processes, which is much simpler than conventional methods, for example, such as that of the Utah array. We demonstrate the density of microneedles can be as high as 625 microneedles $\mathrm{mm}^{-2}$ and the aspect ratio of microneedles can be as high as $\sim 25$. Also, the controllability of microneedle cross-sectional shapes is demonstrated.

\section{Experimental Methods}

\subsection{Fabrication Step Overview, Materials, and Chemicals}

Figure 1 illustrates the fabrication process flow used in the present study for creating microneedle arrays. A four-inch silicon ( $\mathrm{Si}$ ) wafer ( $p$-type $<100>, 525 \mu \mathrm{m}$ in thickness) was used as a processing wafer. Another Si wafer in the same specification was used as a carrier wafer during the 2 nd DRIE step. We used the conventional DRIE Bosch process and the detail conditions of each step are summarized in Table 1. For completely isolated microneedles, a siliconon-insulator (SOI) wafer was used, which had 350, 1, and $100 \mu \mathrm{m}$ in thicknesses for handling $\mathrm{Si}$, buried oxide, and device Si layers, respectively (later in Fig. 8). To have final microneedle arrays, our fabrication processes had one photolithography which was followed by two DRIE steps: (1) photoresist coating on a Si substrate (Fig. 1a), (2) photolithography for patterning the photoresist (Fig. 1b), (3) anisotropic etching of Si using DRIE and photoresist removal (Fig. 1c), (4) bonding to a carrier wafer using a photoresist layer (Fig. 1d), (5) isotropic etching of Si using DRIE (Fig. 1e, f), and (6) removal of the bonding photoresist for release (Fig. 1g). All fabrication steps of the proposed method were performed without any wet etching. Acetone, isopropyl alcohol, and all other solvents were purchased from Dae-Jung Chemicals (Seoul, Republic of Korea).

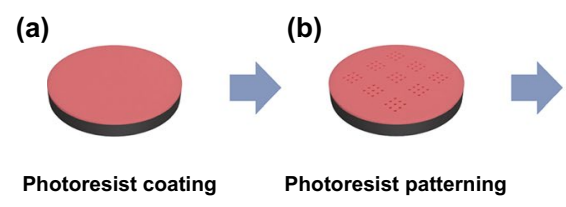

(c)
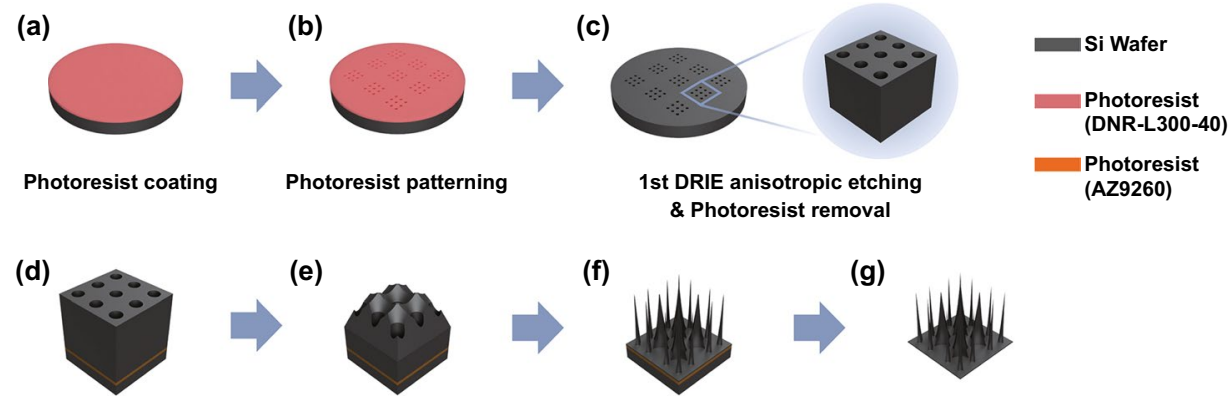

(e)

Bonding of carrier wafer using photoresist

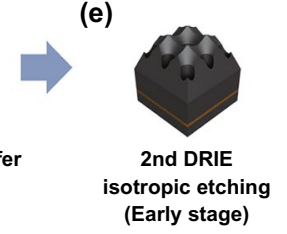

(f)

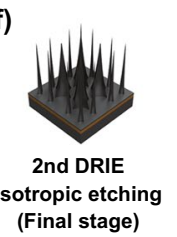

(g)

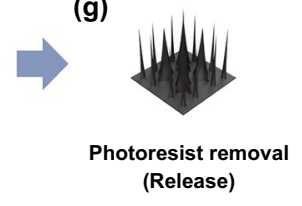

Fig. 1 Schematics of fabrication steps of the microneedle arrays. a Photoresist (DNR-L300-40) coating on a processing Si wafer. b Patterning the photoresist as a masking layer for following deep reactive ion etching (DRIE). c Anisotropic etching (the 1st DRIE) of Si and subsequent removal of the photoresist. Inset shows magnified view of microwells formed by the anisotropic etching. $\mathbf{d}$ Bonding a carrier wafer under the processing wafer using another photoresist (AZ9260). e, f Isotropic etching (the 2nd DRIE) of Si microstructures. The microstructures at early $(e)$ and final $(f)$ stages are shown. $\mathbf{g}$ Release of the fabricated microneedle arrays from the carrier wafer by removing the bonding photoresist

Table 1 Process parameters of deep reactive ion etching for one single DRIE cycle

\begin{tabular}{llll}
\hline & Passivation step & Breakthrough etch step & Main etch step \\
\hline Time (s) & 7 & 9 & 14 \\
Step overlap (s) & 0.5 & 1 & 0 \\
Gas flow ratio of $\mathrm{C}_{4} \mathrm{~F}_{8}: \mathrm{SF}_{6}: \mathrm{O}_{2}$ & $120: 0: 0$ & $25: 130: 13$ & $0: 10: 1$ \\
Pressure (mTorr) & 94 & 94 & 15 \\
RF coil power (W) & 800 & 2500 & 600 \\
RF platen power $(\mathrm{W})$ & 0 & 20 & 0 \\
\hline
\end{tabular}




\subsection{Photolithography and Anisotropic Etching}

DNR-L-300-40 (Dongjin Semichem Inc., Seoul, Republic of Korea) was patterned to serve as a masking photoresist layer for the 1st DRIE step. First, the photoresist (DNRL-300-40) was spin-coated at a thickness of $5.5 \mu \mathrm{m}$ on a processing wafer (Fig. 1a). After soft baking on a hot plate, the coated photoresist was exposed to UV light using a mask aligner (MA6, SÜSS MicroTec SE, Garching, Germany). After post-exposure bake on the hot plate, the processing wafer was soaked in the fresh developer solution, AZ 300 MIF, and then agitated and rinsed with deionized water (Fig. 1b). After the photolithography, deep microwell arrays were formed on the Si substrate by the conventional DRIE processes (Fig. 1c) using an Omega ${ }^{\circledR}$ LPX-DSi Etch system (SPTS Technologies Ltd., Newport, United Kingdom). Then, the masking photoresist was removed (Fig. 1c) using the $\mathrm{O}_{2}$ plasma asher (V15-G, PINK GmbH Thermosysteme, Wertheim, Germany). This anisotropic etching defined shapes and distributions of microwells depending on the photomask designs, which will eventually determine the final geometries of the microneedles. The purpose of the photoresist as a bonding material was to increase isotropic feature during the DRIE process. It is conjectured that the bonding photoresist layer hindered the cooling of the processing wafer [43], as well as diminished the straight movement of biased ion [52-54]. Interestingly, the enhanced isotropic feature during the DRIE process was not observed with the crystal bond which is typically used as bonding materials for effective cooling during DRIE processes.

\subsection{Isotropic Etching with Photoresist Bonding}

The Si structures remained after the anisotropic DRIE were further isotropically etched to form microneedles. After the anisotropic etching, another photoresist AZ 9260 (Merck KGaA, Darmstadt, Germany) was used to bond a carrier wafer underneath the processing wafer for the following 2nd DRIE: a layer of AZ 9260 was spin-coated in $10 \mu \mathrm{m}$ on a bare (carrier) wafer. Then, the processing wafer was bonded onto the carrier wafer on a hot plate at $110^{\circ} \mathrm{C}$ (Fig. 1d). Si structures more isotropically etched during the 2nd DRIE step (Fig. 1e, f). The final microneedle structures were released from the carrier wafer by removing the bonding photoresist
(Fig. 1g). For removing the photoresist layer, AZ 300 MIF (Merck KGaA, Darmstadt, Germany) and acetone were used.

\subsection{Characterization of Microneedles}

Scanning electron microscopy (SEM; Nova Nano SEM 450, FEI, Eindhoven, Netherlands) was performed to measure dimensions of etched microwell arrays after the 1st DRIE, as well as to characterize the structure of microneedle arrays after the 2nd DRIE. Also, to assess the penetration capability of microneedles, insertion forces to agarose gel tissue phantoms and a mouse brain were measured using a digital force gauge (M5-012E, Mark-10, Copiague, NY, USA) placed on a motorized test stand (ESM303E, Mark-10). The agarose gel phantoms were prepared in two different concentrations $(0.5 \%$ and $1 \%)$ by mixing agarose powder (Sigma-Aldrich, St. Louis, MO, USA) in a phosphate buffered solution. It has been known that $\sim 0.5 \%$ agarose gel mimics the compression mechanics of the brain [36] but we also doubled concentration (1\%) of agarose gel to additionally verify the penetration capability of microneedles to a harder sample.

An adult mouse (C57BL/6 strain, 8 weeks old) was anesthetized by intraperitoneal injection of urethane $\left(1.5 \mathrm{~g} \mathrm{~kg}^{-1}\right)$ and then euthanized by cervical dislocation for the acute experiment. The middle of the mouse head was shaved, and its skull was drilled to expose the brain surface. The animal experiment protocol was approved (KIST-2020-166) by Institutional Animal Care and Use Committees of KIST.

\subsection{Transfer Molding of Microneedles}

To demonstrate a feasibility of creating microneedles in different materials, we used an array of Si microneedles as the first mold and a polydimethylsiloxane (PDMS; Dow Corning Corporation, Midland, MI, USA) layer as the second mold (as shown later in Fig. 12). First, the surface of microneedles was coated with $50 \mu \mathrm{L}$ trichloro $(1 \mathrm{H}, 1 \mathrm{H}, 2 \mathrm{H}, 2 \mathrm{H}$-perfluorooctyl) silane (PFOTS, Sigma-Aldrich, St. Louis, MO, USA) for $2 \mathrm{~h}$ inside a vacuum desiccator to promote later release between the Si microneedles and the PDMS layer. Next, the PDMS, which was mixed with a curing agent at a ratio of 10:1, was poured onto the microneedles and cured for $2 \mathrm{~h}$ at $80{ }^{\circ} \mathrm{C}$. Subsequently, a solution of $7 \%$ silk fibroin 
was poured onto the prepared PDMS mold. Finally, the silk microneedle array was peeled off from the PDMS mold.

The silk fibroin solution was prepared as follows: (1) a silkworm cocoon (13.75 g; Uljinsilk, Gyeongsangbuk-do, Republic of Korea), sodium oleate (1.03 g), sodium carbonate $(0.69 \mathrm{~g})$ were heated with distilled water $(350 \mathrm{~mL}),(2)$ after $1 \mathrm{~h}$, the mixture was filtered and washed several times with distilled water, (3) the filtered mixture was heated again with $700 \mathrm{~mL}$ of distilled water for $1 \mathrm{~h}$ and stored overnight at room temperature, (4) a chemical reactor containing the dried mixture and $120 \mathrm{~mL}$ of lithium bromide (LiBr) was placed in a $70{ }^{\circ} \mathrm{C}$ water bath for $1 \mathrm{~h}$ with an overhead stirrer rotating at $300 \mathrm{rpm}$, (5) the silk mixture was dialyzed for 2 days, and (6) the dialysis was verified by using a pocket salt meter and the mixture filtered using a Kimtech wipe and stored overnight at room temperature. All chemicals were used as purchased without further alteration.

\section{Results and Discussion}

\subsection{Anisotropic Etching Creates Microwell Structures}

The control of microneedle heights would be essential to make microneedles adequately reach target depths $[2,55]$.
In our fabrication approaches, the height control begins with the 1st anisotropic DRIE step. In Fig. 2a, photomask patterns which determined microwell areas to be etched are shown in white circles with black solid lines. After the 1st DRIE process, the cross section of resulting deep microwells was imaged and their depths were measured in the SEM image (Fig. 2b). To further accurately characterize our DRIE process, we plotted etching depths as a function of DRIE cycles (Fig. 2c). For all microwell diameters $(d)$ we tested, the depth of etched microwells increased linearly as the number of DRIE cycles increased. Also, consistent with a previous study [56], it appears that a bigger opening in the masking photoresist layer resulted in a deeper trench at a given the number of DRIE cycles. It is thought to be because, due to the higher gas accessibility, the anisotropic DRIE etched Si faster in bigger microwells [57]. For example, in case of the microwell diameter of $100 \mu \mathrm{m}$, the etched depth increased from 149 to $388 \mu \mathrm{m}$ with increasing DRIE cycles from 200 to 650 (darkest blue trace of Fig. 2c). In contrast, in case of the microwell diameter of $50 \mu \mathrm{m}$, the etched depth increased from 131 to $313 \mu \mathrm{m}$ (light blue trace of Fig. 2c). In addition, etch rate difference was more clearly shown for greater numbers of etching cycles: When the (a)
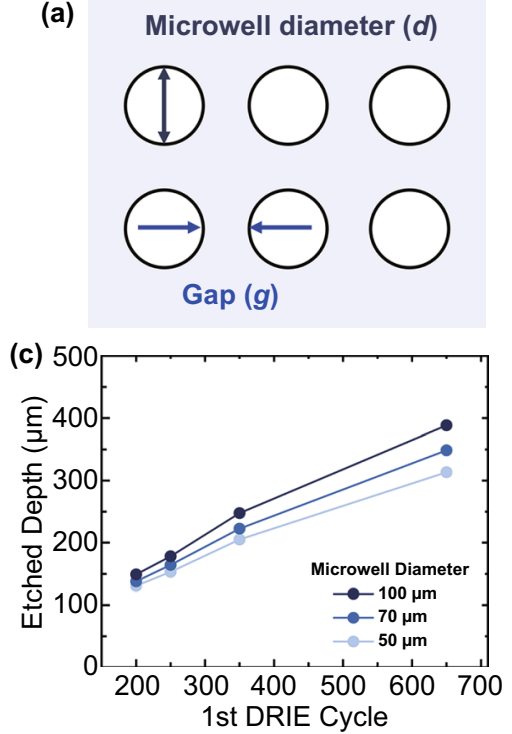

(b)

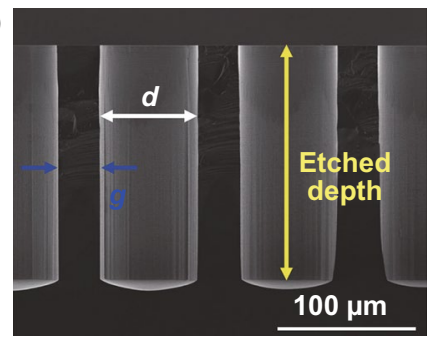

(d)

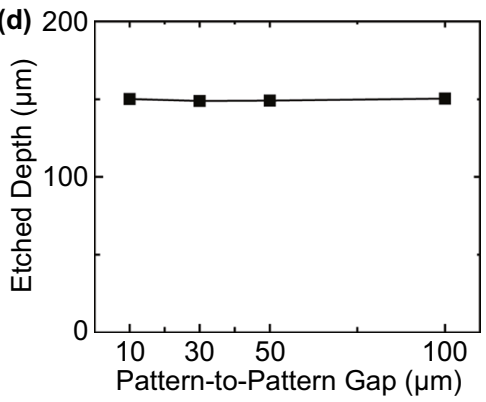

Fig. 2 Characterization of the anisotropic etching (the 1st DRIE) process to create microwell structures a Microwell diameter (d) and pattern-topattern gap $(g)$ are shown as design parameters of a photomask. b A SEM image shows cross-sectional view of the microwells fabricated by 250 cycles of anisotropic etching $(d=70 \mu \mathrm{m}$ and $g=30 \mu \mathrm{m})$. $\mathbf{c}$ Etched depths of microwells are plotted as a function of the 1st DRIE cycle numbers for 50,70, and $100 \mu \mathrm{m}$ in microwell diameter. $\mathbf{d}$ Depth of microwells etched by 250 DRIE cycles as a function of pattern-to-pattern gaps ranging from 10 to $100 \mu \mathrm{m}$. Microwell diameter was fixed at $50 \mu \mathrm{m}$ 
diameter of microwell increased from 50 to $100 \mu \mathrm{m}, 200$ cycles etched $13 \%$ (from 131 to $149 \mu \mathrm{m}$ ) deeper microwells, while 650 cycles etched $23 \%$ (from 313 to $388 \mu \mathrm{m}$ ) deeper ones (compare lightest vs. darkest blue symbols in Fig. 2c). Interestingly, the pattern-to-pattern gap ( $g$ ) did not show any substantial change on the etched depth of microwells (Fig. 2d). Our results suggest that low-density microneedle arrays which have larger etching areas would require smaller numbers of DRIE cycles.

\subsection{Additional Isotropic Etching Produces Microneedle Structures}

To create sharp microneedles at the area surrounded by neighboring four microwells, we performed another DRIE step which was designed to promote isotropic etching of the Si layer by removing the masking photoresist and using another photoresist to reduce substrate cooling (see Sect. 2). Figure 3 shows how the silicon structures gradually changed as the isotropic etching progressed, eventually becoming the sharp microneedles. Due to the enhanced lateral etching, the early stage of isotropic etching widened the microwells (Fig. 3b, bi, and bii). When the adjacent widening microwells touched each other, those interfacial areas were further vertically etched to form bridged valleys (Fig. 3c, d, di, and dii). As the isotropic etching continued, the heights of bridged valleys decreased and the blunt tips of microneedles were sharpened (compare Fig. 3dii vs. fii).

Next, we examined the effects of a couple of photomask design parameters on the geometries of final microneedle structures after both DRIE steps (Fig. 4). First, we compared microneedles created from three different microwell diameters (d), while the pattern-to-pattern gap ( $g$ ) was fixed at $30 \mu \mathrm{m}$ (Fig. 4a). Gray areas with dashed lines of Fig. 4a represent

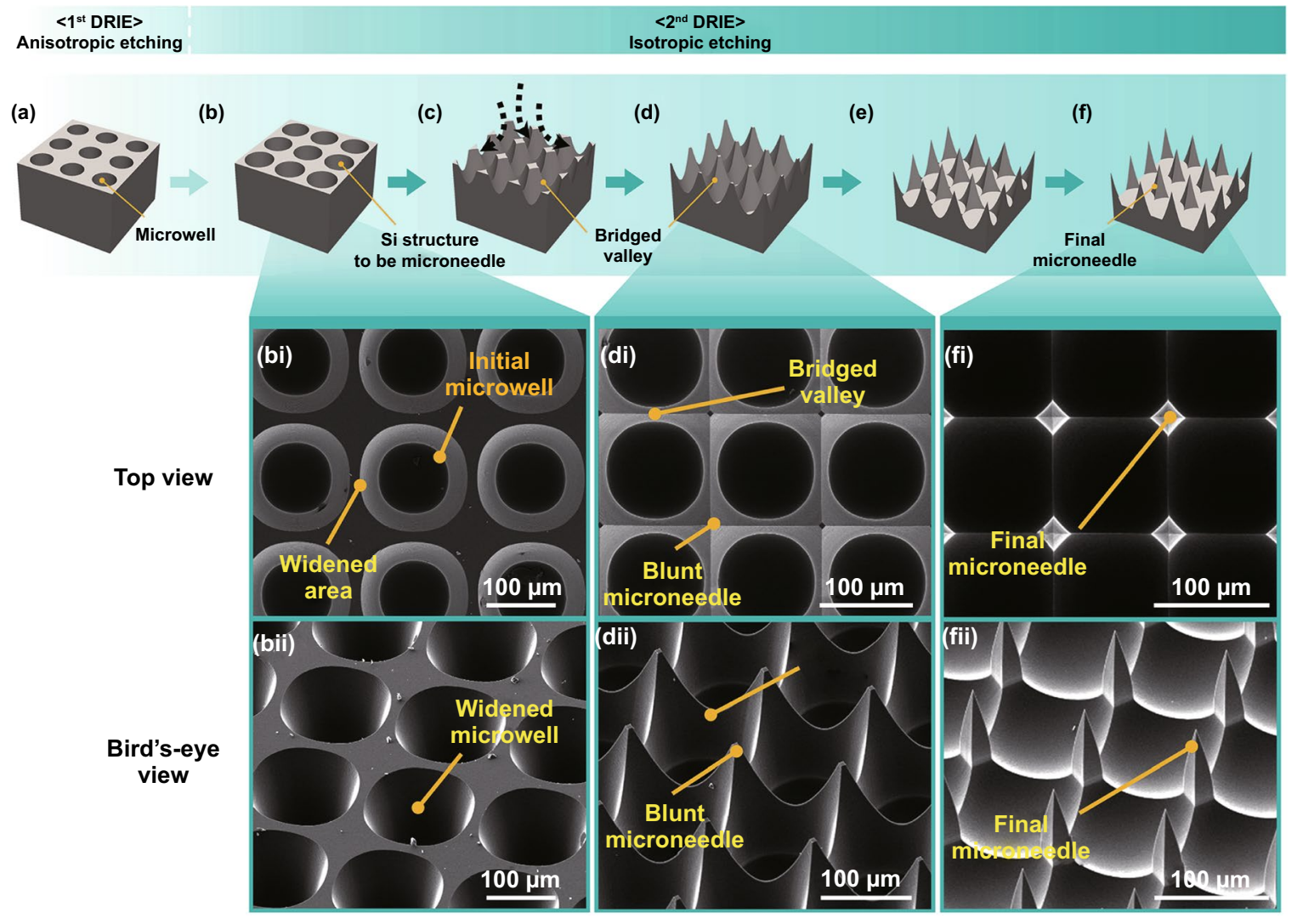

Fig. 3 Schematics and SEM images of Si geometries during the isotropic etching (the 2nd DRIE) process to sharpen microneedles. a Fabricated microwells after the 1st DRIE step. b Early stage of the 2nd DRIE: Initial microwells are widened due to isotropic etching. c, d Intermediate stage: Adjacent widened microwells connected to each other, forming blunt microneedles and bridged valleys. e, f Final stage: Sharp microneedles are produced and the bridged valleys are gradually disappeared. SEM images in top view and bird's-eye view are shown at the bottom of schematics for early $(b i, b i i)$, intermediated $(d i, d i i)$, and final $(f i, f i)$ stages 
widening parts of microwells during the 2nd isotropic DRIE step; this widening eventually created microneedles in the areas highlighted in blue. For the photomask patterns with all three diameters, we performed the same numbers of $1 \mathrm{st}$ and 2nd DRIE cycles (250 and 150, respectively), and then, we obtained longer microneedles from bigger microwells (Fig. 4b). This unique feature (i.e., microneedle height controllability by varying microwell diameters) enabled us to monolithically incorporate microneedles in various heights even with identical DRIE processes (see Sect. 3.7 and Fig. 6). Quantitatively, the final heights of microneedles were 56, 79, and $115 \mu \mathrm{m}$ from microwells of 50, 70, and $100 \mu \mathrm{m}$ in diameter, respectively (black trace of Fig. 4c). It seems like there were two factors that come into play: (1) earlier anisotropic etching was faster in wider microwells (see Fig. 2d and green curve of Fig. 4c), and (2) the relatively smaller size of micropillars was over-etched than bigger ones during the isotropic DRIE (black curve of Fig. 4c). Regarding the second factor, in (a) (i)

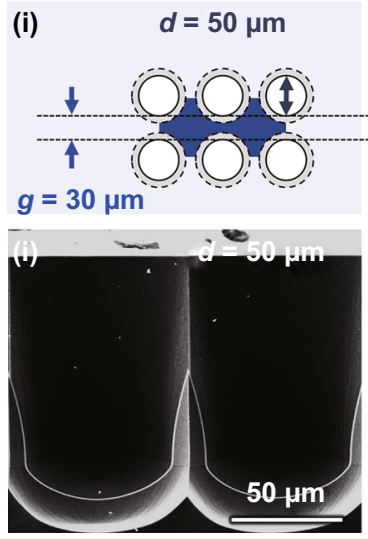

(c)

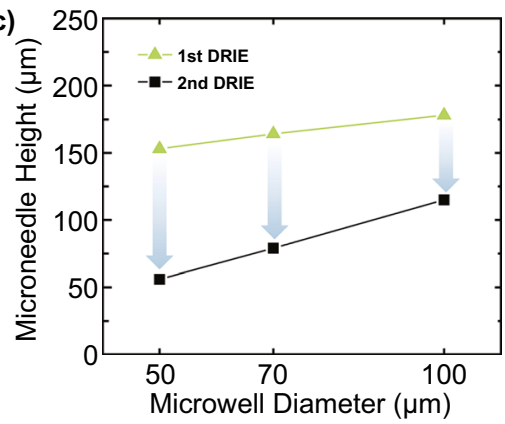

(e)

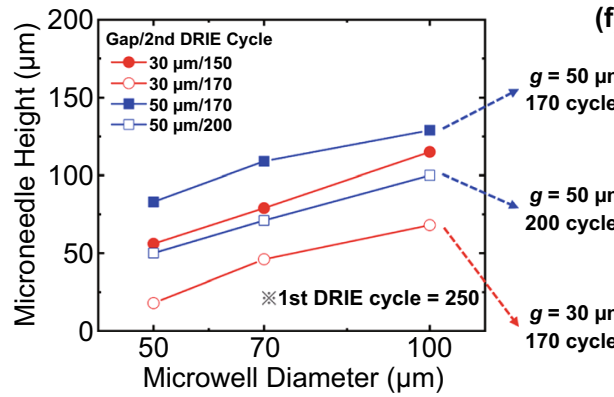

(ii) $d=70 \mu \mathrm{m}$

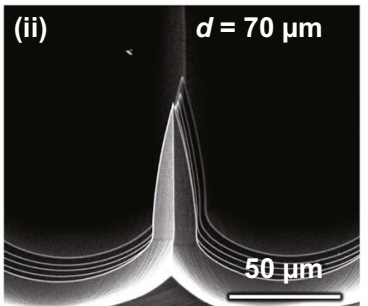

(iii) $d=100 \mu \mathrm{m}$

(d)

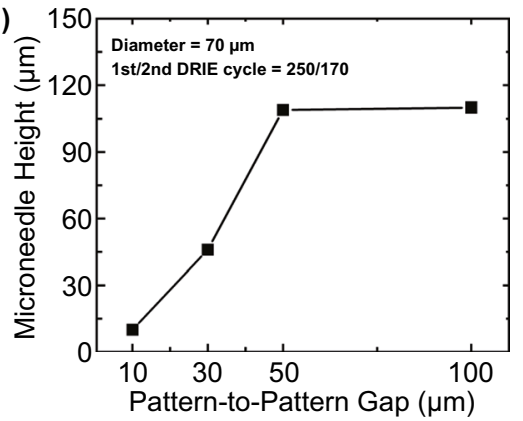

(f)

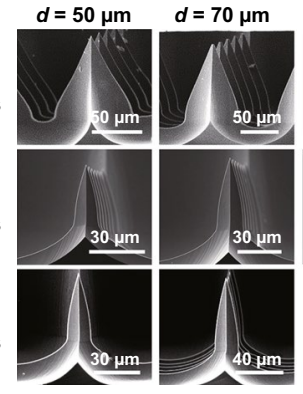

$d=100 \mu \mathrm{m}$

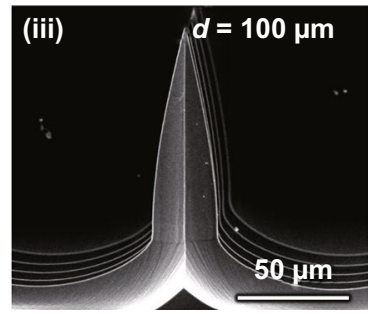

$d=100 \mu \mathrm{m}$

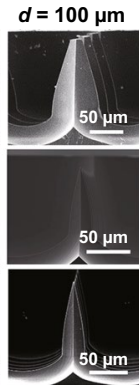

Fig. 4 Effects of the design parameters and cycles of isotropic etching on final microneedle structure. a Illustration of photomask designs with a fixed gap $(g)$ of $30 \mu \mathrm{m}$ and microwell diameters $(d)$ of 50, 70, and $100 \mu \mathrm{m}$. Solid and dashed circles indicate initial microwell patterns after the anisotropic DRIE and widened areas during the isotropic DRIE, respectively. Blue polygons represent Si areas to be microneedles. b SEM images showing cross-sectional views of microneedles created from the three microwell diameters. $\mathbf{c}$ Microneedle heights as a function of different microwell diameters after the 1st and the 2nd DRIEs. d Microneedle heights as a function of pattern-to-pattern gap. e Microneedle heights as function of microwell diameters for several combinations of pattern-to-pattern gap and number of the 2nd DRIE cycles. f SEM images of microneedles fabricated from the three conditions described in panel $e$. Note that, scale bars are different in each SEM image 
case of $100-\mu \mathrm{m}$-wide microwells, for example, the 150 cycles of isotropic etching process were proper to fabricate long and sharp microneedles; for 50- $\mu \mathrm{m}$-wide microwells, however, the same number of DRIE cycles shortened the microneedles by design to have different lengths of microneedles nearby even after enough sharpening (compare Fig. 4biii and 4bi). Given the fact that 250 cycles of the 1 st DRIE did not make big differences in etching depth (see green line in Fig. 4c), the aforementioned second factor seems more critical in making microneedle height differences dependent on the microwell diameter (see black line in Fig. 4c).

Different from the anisotropic etching (Fig. 2e), the pattern-to-pattern gap affected the microneedle height during the isotropic etching (Fig. 4d): the microneedle height increased up to $50 \mu \mathrm{m}$ of the gap, but the height was saturated beyond that point. These results were also caused by the over-etching effect due to the different thicknesses (or widths from the top view) of remaining micropillars after the 1st DRIE step: Smaller gaps created narrower micropillars which were also vertically etched faster. Then, we also varied both patternto-pattern gaps and numbers of 2nd DRIE cycles but with the fixed 1st DRIE cycles of 250 (Fig. 4e). First, when we increased the 2nd DRIE cycles from 150 to 170 for microwell patterns separated by $30 \mu \mathrm{m}$ gaps, microneedles heights were substantially reduced for all microwell sizes (compare red lines in Fig. 4e). For instance, the microneedle height created from $100-\mu \mathrm{m}$-wide microwells decreased significantly from 115 to $68 \mu \mathrm{m}$ because of the over-etching effect. Similarly, the pattern-to-pattern gap of $50 \mu \mathrm{m}$ also decreased the microneedle height with increased isotropic etching cycles (compare blue lines in Fig. 4e). However, this reduction in height is unavoidable to obtain the sharp microneedle. The final microneedle structures obtained from different conditions were summarized in a series of SEM images (Fig. 4f): If the pattern-to-pattern gap was increased from 30 to $50 \mu \mathrm{m}$ with 170 cycles of isotropic etching, the sharpness of microneedles decreased along with increasing their microwell diameter size. For example, in the first row of Fig. $4 \mathrm{f}$, the microneedle from $100 \mu \mathrm{m}$ microwell shows a dull shape compared to other 50 and $70 \mu \mathrm{m}$ microneedles. It seems like these longer but dull microneedles were resulted from insufficient isotropic etching. Accordingly, when the 2nd DRIE cycle of the $50 \mu \mathrm{m}$-gap microwells was increased to 200 , the microneedles became sharper (SEM images shown in the second row of Fig. 4f). It is interesting to note that both overall shape and sidewall profiles of those microneedles were remarkably similar to the microneedles which were fabricated from $30 \mu \mathrm{m}$-gap microwells and isotropically etched during 170 cycles (compare SEM images shown in the second and third rows of Fig. 4f). Taken together, our results suggest that the isotropic etching process initially contributed a way of sharpening the microneedles, but it rather decreased the height of microneedles after a certain number of cycles depending on pattern-to-pattern gaps. For more optimization of fabrication processes to precisely adjust the target height and shape of the microneedle, a comprehensive understanding of the effects of the microwell diameter, the 1st/2nd DRIE cycles, and the pattern-to-pattern gap would be needed in addition to our results described above.

\subsection{High-Aspect Ratio Microneedles Using Dumbbell Well Photomask Patterns}

Although the aspect ratio of the fabricated microneedles can be modulated by varying design and process parameters (Fig. 4), the achievable aspect ratio of the microneedles using the circular microwell array was pretty low $(\sim 3.2)$. It was thought to be because of the bridged valley formed between neighboring microneedles (Fig. 3dii), which should be minimized during the 2nd DRIE process; however, the continuous isotropic etching resulted in shorter microneedles, reducing their aspect ratio. Increasing diameters of microwells can enhance the aspect ratio but make pitches bigger, lowering the density of the microneedle array. To achieve both highaspect ratio and high density of microneedles, we devised a dumbbell-shaped microwell photomask pattern (Fig. 5a). We connected adjacent microwell patterns to each other to make microneedle-to-be areas isolated even from the first anisotropic etching (blue area; Fig. 5a). After the 1st DRIE (350 cycles), which created the micropillars (Fig. 5bi), the subsequent 2 nd DRIE (50 cycles) turned the individual micropillars into slender microneedles with sharp tips (Fig. 5bii). The SEM images in Fig. 5c-e show the representative microneedles fabricated after 350 and 50 cycles of the 1 st and 2nd DRIEs for the different diameters of microwells. Due to the different microwell sizes, the microneedle base width was decreased from 16 to $8 \mu \mathrm{m}$, while the microwell diameter changed from 75 and $150 \mu \mathrm{m}$. Also, the same isotropic etching did not introduce any further sharpness for the microwells of $75 \mu \mathrm{m}$ in diameter (Fig. 5ciii), while dull and extremely sharp microneedles were fabricated from the microwells of 90 and $150 \mu \mathrm{m}$, respectively (Fig. 5diii and eiii). Because the 
(ai)
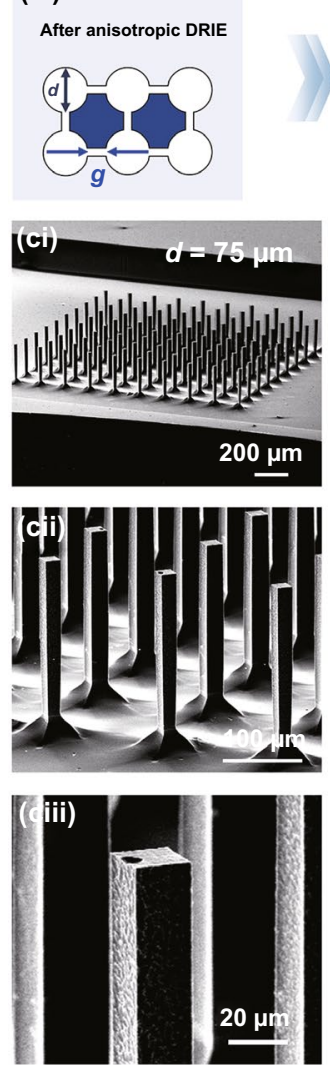

(aii)

After isotropic DRIE
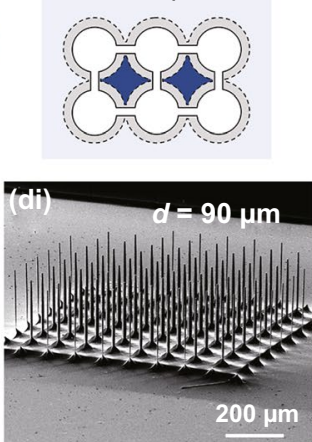

200. $\mathrm{nm}$

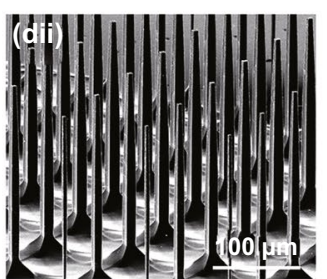

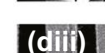

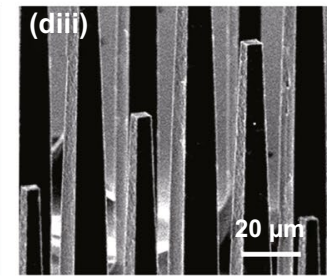

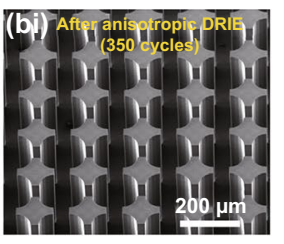
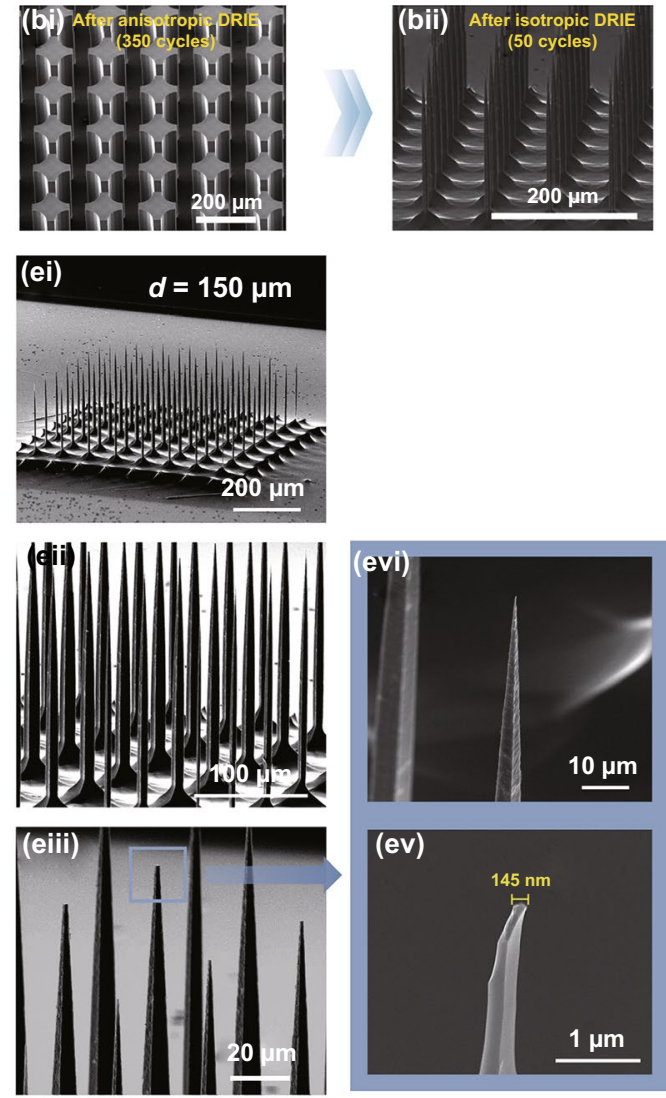

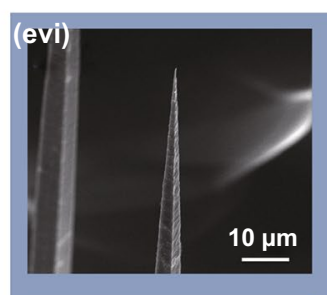

(ev)

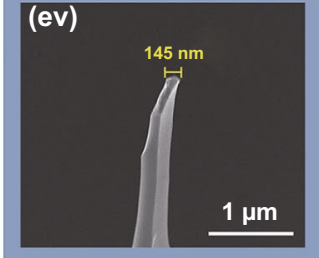

Fig. 5 Fabrication of high-aspect ratio microneedles using dumbbell well photomask patterns. a Illustration of dumbbell well pattern designs after anisotropic and isotropic DRIE steps. b SEM images of anisotropic (350 cycles) and isotropic (50 cycles) process. c-e SEM images of microneedles created from dumbbell diameters of 75, 90, and $150 \mu \mathrm{m}$. Enlarged SEM images show the tip of microneedles from the $150-\mu \mathrm{m}$-wide dumbbell well pattern in panel $e(e v i$ and $e v)$. The width of microneedle tip was about $145 \mathrm{~nm}$

dumbbell well patterns did not produce any bridge valleys much smaller, number of 2nd DRIE cycles was enough to isolate microneedles, minimizing over-etching. As a result, the highest aspect ratio $(\sim 25)$ was obtained from the dumbbell well diameter of $150 \mu \mathrm{m}$. Also, those microneedles were remarkably slender, demonstrating the ratio between base and top widths of $\sim 1$.9. The magnified SEM image also reveals extremely sharp sub-micron $(\sim 145$-nm-wide) tip at the very end of microneedles (Fig. 5evi and ev).

\subsection{Microneedles with Irregular Height Distribution}

In neuro-engineering applications, an array of microneedle electrodes in various length can enable researchers access neural information processed in both vertical and horizontal directions [58]. However, it has been challenging to monolithically incorporate out-of-plane microneedles (e.g., Utah probe) of different heights (or lengths) although convoluted [30] or slanted [23] electrode arrays had been demonstrated. To cover variable penetration depths in 3D neural tissues such as cortex, retina, and nerve fiber, it would be necessary to monolithically integrate microneedles in various heights in a precisely designed manner.

In Sect. 3.2, we demonstrated the microneedle length modulation as a function of the microwell diameter designed in photomask (Fig. 4). With this unique feature of our proposed method, diverse heights of microneedles were obtained in a single $\mathrm{Si}$ wafer using identical fabrication processes but different photomask designs, which have various diameters and distributions of dumbbell wells (Fig. 6). First, to have convex shape of the surface defined by microelectrode tips, we placed $30 \mu \mathrm{m}$ of small dumbbell wells at the center of the photomask and made the diameters of wells gradually 

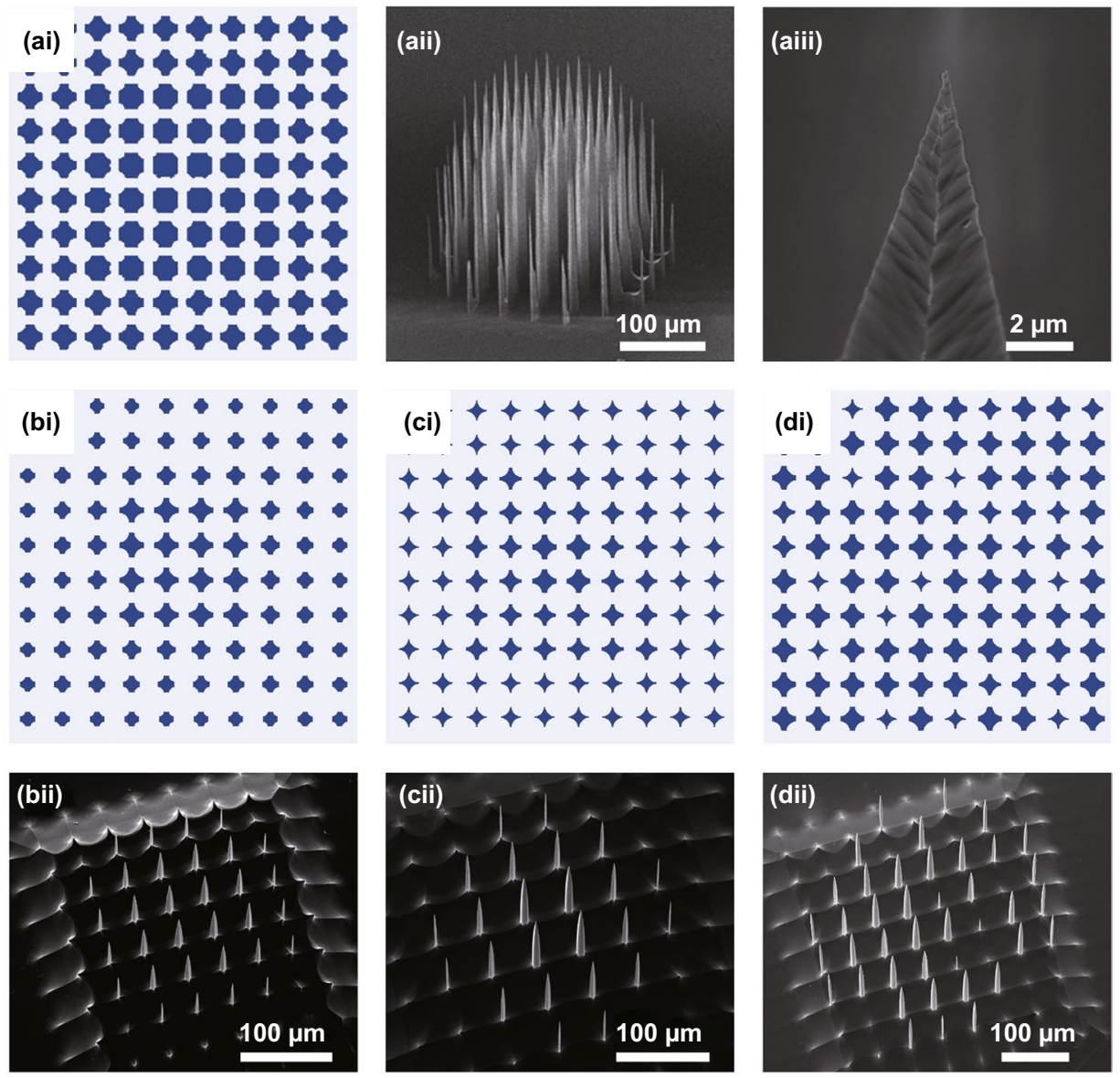

Fig. 6 Monolithic fabrication of microneedles in various height distributions on a single wafer. a Photomask design for a convex microneedle array (ai) and SEM images of the fabricated convex microneedle array (aii and aiii). b-d Photomask design for shorter (bi) and lower density (ci) convex microneedle arrays, and microneedles with an irregular height distribution (di). SEM images of the fabricated microneedle arrays (bii-dii) from those photomask designs

increased up to $40 \mu \mathrm{m}$ with decreasing gap from 20 to $10 \mu \mathrm{m}$ as the photomask pattern moved outward (Fig. 6ai). Indeed, the fabricated microneedle array had a convex tip contour with longer microneedles located at the center and shorter microneedles at the perimeter of the sample (Fig. 6aii). In this photomask design, the microneedle heights ranged from 68.54 to $246.44 \mu \mathrm{m}$, resulting in the biggest difference of $177.90 \mu \mathrm{m}$. Theoretically, the height deviation across microneedles in a given array can be further increased by using thicker processing wafers. A magnified view of SEM image clearly shows the apex of the microneedle has a sub-micron tip (Fig. 6aiii).

To show other examples for diverse microneedle profiles, we designed photomasks that have different dumbbell well diameters and pattern-to-pattern gaps (Fig. 6bi, ci, and di) and fabricated microneedle arrays using those photomasks (Fig. 6bii, cii, and dii). For instance, in case of Fig. 6bi and ci, we increased the diameters and decreased gap of dumbbell wells than those of Fig. 6ai, creating shorter and low-density convex shape microneedles (compare Fig. 6bii and cii vs. Fig. 6aii). When the pattern-to-pattern gap was changed irregularly (Fig. 6di), microneedles with irregular heights were produced (Fig. 6dii). These examples suggest a possibility that our proposed method can be further expanded to fabricate microneedle arrays which have other height distributions in a designed manner.

\subsection{High-Density and Completely Isolated Microneedle Arrays}

Another important design feature of the microneedle array is density. As a penetrating neural interface, higher density 
microneedle would access densely populated neurons or nerve fibers individually, resulting in a more sophisticated investigation of the mechanism underlying complex spatial neural interactions. To explore whether this high density is achievable, we designed dumbbell well arrays that have three different combinations of microwell diameters and gaps (Fig. 7a-c). Figure $7 d$ shows microneedle arrays fabricated in the highest density from the same photomask pattern with Fig. 7c: It has 15,625 microneedles in $5 \times 5 \mathrm{~mm}^{2}$ of a $\mathrm{Si}$ wafer (Fig. 7di), corresponding to the microneedle density of 625 microneedles $\mathrm{mm}^{-2}$. The final microneedle structures were also extremely uniform across the array (Fig. 7dii) and had remarkably sharp tips (Fig. 7diii). In contrast to wet etching processes which showed considerable variations in microneedle lengths [23], our proposed method using DRIEs demonstrated $<\sim 1.2 \%$ variations from target heights. Also, the width of microneedles at $90 \%$ of the total length from the bottom varies $\sim 1.4 \%$ at most, further supporting the good uniformity of our fabrication method. We further confirmed that these high-density microneedle arrays could be successfully fabricated with all three kinds of different dumbbell well diameters (Fig. 7). Given the length of the microneedles, the high-density sharp microneedles shown in Fig. 7 might be a good candidate for intra-retinal stimulation [59] to differentially activate $\mathrm{ON}$ and OFF pathways of the retina $[60,61]$.

We observed that bulk Si wafers left small hillocks at the base of microneedles (Fig. 5). A silicon-on-insulator (SOI) wafer can be used to create a flat microneedle base. Similar to the fabrication processes for bulk Si wafers, we first patterned a photoresist layer (first row of Fig. 8a) but we used the SOI wafer upside down to etch the handle layer to obtain microneedles as long as possible. The main advantage of the use of SOI wafers appeared during the 1st DRIE step (second row of Fig. 8a): We were able to over-etch the top Si layer ( 700 cycles for $350 \mu \mathrm{m}$ for the SOI wafer we used) since the oxide layer acted as a good etch stop layer without damaging the bottom Si layer. Finally, individual microneedles which had a precise height same as the thickness of Top Si layer were created. Then, after the masking photoresist removal, a carrier wafer was bonded under the SOI wafer. Lastly, 50 cycles of the DRIE process were performed for isotropic etching (third row of Fig. 8a). The SEM image of the final microneedles showed neither bridge valleys nor hillocks at the microneedle bases (compare Fig. 5cii and 8b).

\subsection{Insertion Force Analysis}

To evaluate the feasibility of practical use of the fabricated sharp microneedles, we measured the required force to insert microneedles into tissue phantoms and a mouse brain. As a reference, the smallest insertion force of microneedles to human skin reported so far is $10 \mathrm{mN}$ [48], which is still 500 times bigger than the force used by a mosquito to insert its labium $(18 \mu \mathrm{N})$ [62]. With the microneedles fabricated from SOI wafers which had approximately $\sim 25$ and $\sim 10 \mu \mathrm{m}$ in
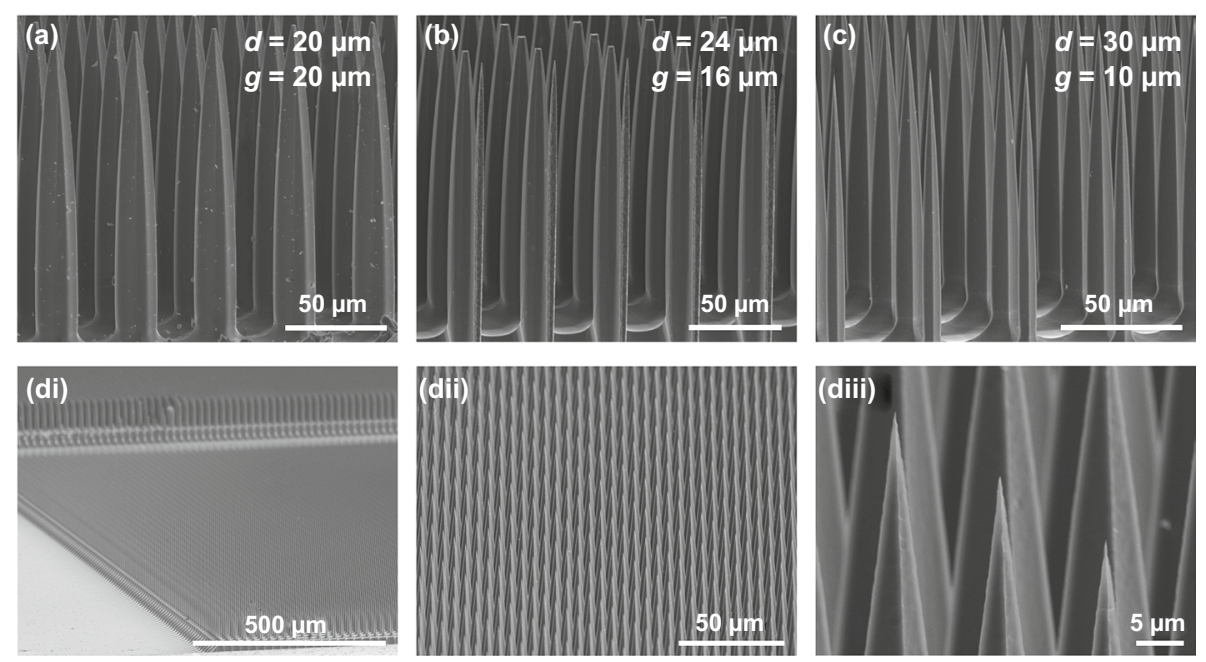

Fig. 7 High-density microneedle arrays using the dumbbell well patterns. a-c SEM images of final microneedle structures fabricated from different design parameters. Dumbbell well diameter $(d)$ and pattern-to-pattern gap $(g)$ are shown at the top right. d SEM images of the high-density microneedle array (di-dii) and enlarged view showing sharp tip (diii). The design parameters were same as panel $c(d=30 \mu \mathrm{m}, g=10 \mu \mathrm{m})$ 
(a) Photoresist Patterning

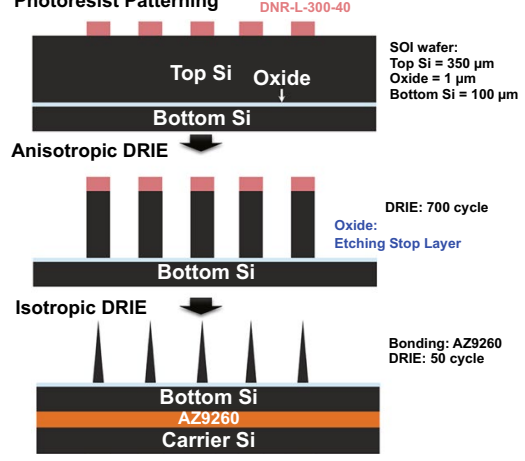

(b)

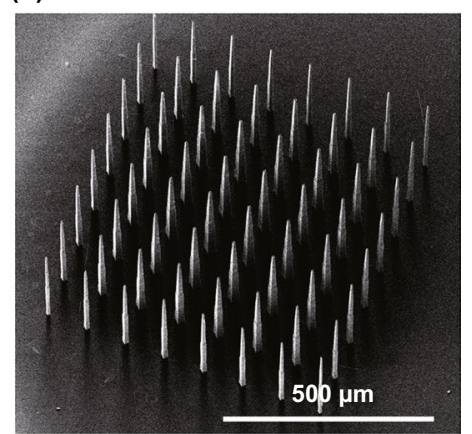

Fig. 8 a Fabrication process flows using a silicon-on-insulator (SOI) wafer for complete isolation of microneedles. The oxide layer of SOI wafer acts as an etching stop layer during anisotropic etching to eliminate bridge valleys which appear with bulk Si wafers (see Fig. 3). b SEM image of a final microneedle array fabricated with a SOI wafer

diameters (created from a gap of 30 and $20 \mu \mathrm{m}$, respectively; Fig. 9b, c), the insertion forces were characterized quantitatively (see Sect. 2). Figure 9a shows the measured forces as a function of displacement when microneedle arrays were being inserted to $1.0 \%$ or $0.5 \%$ agarose gels at a speed of $10 \mu \mathrm{m} \mathrm{s}^{-1}$ (Fig. 9d, e). During pre-penetration insertion stage, the microneedles contacted the surface of agarose gels and the monotonic increments of the measured forces were observed until the penetration occurred as highlighted with arrows in Fig. 9a. After the peak point of each plot which shows the different puncture force and displacement, the force was suddenly dropped, indicating post-penetration relaxation stage [62]. As aforementioned, the sharpness of the microneedle is correlated with the design parameter such as the gap of the microwell array (Fig. 4d); the tips of the microneedles with the $20 \mu \mathrm{m}$ gaps were $\sim 1.72 \mu \mathrm{m}$ which were $\sim 6$ times sharper than those with the $30 \mu \mathrm{m}$ gaps (Fig. 9b, c). It is worth noting that the insertion force was smaller for the sharper microneedles (compare red and blue traces in Fig. 9a): When inserting a microneedle array fabricated with the $30 \mu \mathrm{m}$ gaps into the $1.0 \%$ agarose gel, the penetration force was about $6 \mathrm{mN}$. On the other hand, when inserting a microneedle array fabricated with the $20 \mu \mathrm{m}$ gaps into the same agarose gel, it required a $\sim 21 \%$ lower force. These results clearly indicate that the sharper tip of microneedle arrays requires less insertion force, which is consistent with previous work [36]. When inserting the sharper microneedle array into this softer tissue phantom ( $0.5 \%$ agarose gel), the array could be inserted with less insertion force which was about $1.4 \mathrm{mN}$ (light blue trace in Fig. 9a).

As previously reported, since the agarose gel cannot completely replace real biological tissue [36], we also tested the (a)

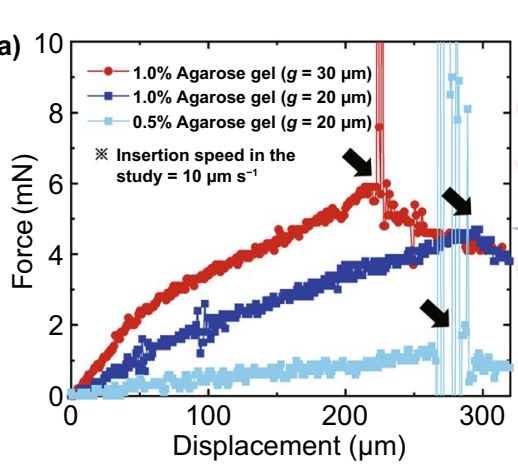

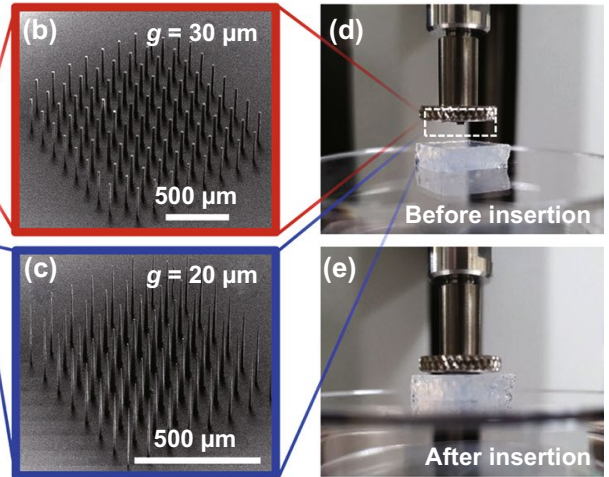

Fig. 9 Insertion tests of the fabricated microneedle arrays to agarose gels mimicking brain tissues. a Force-displacement response during the insertion of microneedle arrays into $0.5 \%$ and $1 \%$ agarose gels at an insertion rate of $10 \mu \mathrm{m} \mathrm{s}^{-1}$. b, $\mathbf{c}$ SEM images of the microneedle arrays used in the insertion tests, which were fabricated from 30 and $20 \mu \mathrm{m}$ gap sizes (for $b$ and $c$, respectively). d, e Photographs of the experiments taken before $(d)$ and after $(e)$ insertion of a microneedle array 
fabricated $10 \times 10$ microneedles array using a mouse brain (Fig. 10a, b). The mouse brain was exposed by removing skin and drilling the skull at a bregma position, but the dura mater was not removed (Fig. 10c). The experiment was conducted while the mouse head was fixed under the digital force gauge (see Experimental Methods). In Fig. 10d, we plotted measured forces as a function of loading displacement for both dull and sharp microneedle arrays shown in Fig. 9b, c. During the dull microneedles compressed the surface of the dura mater with up to $\sim 150 \mu \mathrm{m}$ displacement before puncture $\left(d_{\mathrm{p} 1}\right)$, the force was linearly increased (pre-penetration dimpling stage caused by insertion force; red trace of Fig. 10d). At the moment of penetrating the dura mater, the puncture force $\left(F_{\mathrm{p} 1}\right)$ was measured to be $\sim 25.2 \mathrm{mN}$, which is corresponding to $252 \mu \mathrm{N}$ per one microneedle. This result indicates that even the slightly dull 100 microneedles needed $\sim 10 \mathrm{mN}$ smaller insertion force than that of one single Si microprobe inserted to a rat brain $(\sim 35 \mathrm{mN})$ [63], demonstrating a good penetration capability of our microneedle arrays. As expected from our previous experiment using the agarose gels, the array of sharper microneedles (which is created with pattern gap of $20 \mu \mathrm{m}$ ) needed about $\sim 32 \%$ smaller insertion force to penetrate the dura mater than the dull microneedles. In detail, the sharper microneedles showed a lower insertion force: The puncture force $\left(F_{\mathrm{p} 2}\right)$ was measured to be $\sim 17.2 \mathrm{mN}$, which is corresponding to $172 \mu \mathrm{N}$ per each microneedle (blue trace of Fig. 10d). This insertion force of the sharp individual microneedle is comparable to that of a caterpillar spine to penetrate the mouse skin $(\sim 173 \mu \mathrm{N})$ [62]. Also, compared to the neural probe inserted to a rat brain [63], each sharp microneedle we fabricated required just about one two-hundredth (i.e., $\sim 0.5 \%$ ) force. In both arrays of microneedles, after the puncture of the dura mater, the force was rapidly decreased (postpenetration relaxation stage; Fig. 10d), similar to the measurements performed in the agarose gels (Fig. 9a). Interestingly, the insertion force of the sharp microneedles was more sophisticated, showing less monotonic increment including small bump at $\sim 50 \mu \mathrm{m}$ of displacement. The discrepancy between the two force profiles is highly likely due to the different sharpness of the microneedle arrays. For instance, as
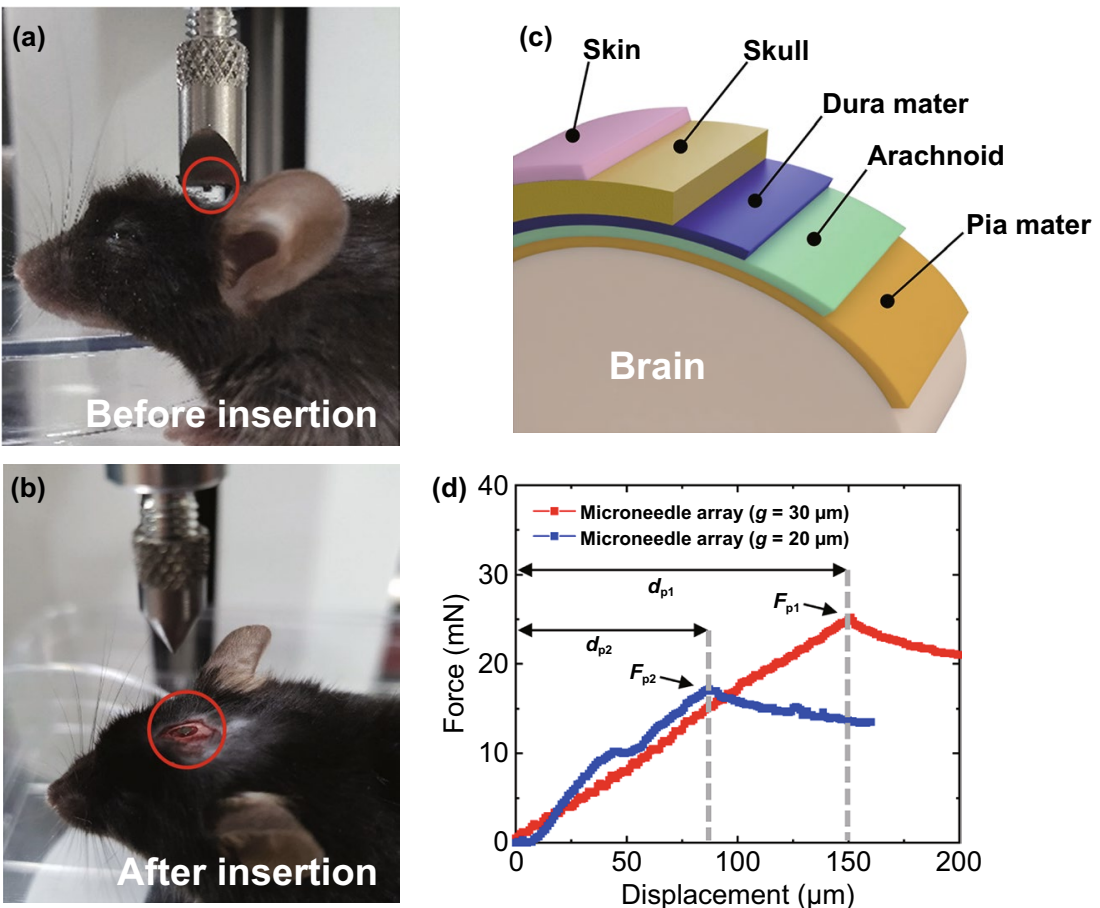

Fig. 10 Insertion tests of the fabricated microneedle arrays to a mouse brain. a, b Microneedle array was positioned above the bregma and completely penetrated the brain. A microneedle array is shown in a red circle in both panels. c Schematic diagram showing layers of a mouse brain. In this experiment, skin and skull were removed but other layers were remained. d Force-displacement plot during the insertion of microneedle arrays with a speed of $10 \mu \mathrm{m} \mathrm{s}^{-1}$ into a mouse brain. We used the two microneedle arrays shown in Fig. $9 \mathrm{~b}$, c: the red and the blue traces show measured forces as a function of loading displacement for dull and sharp microneedles, $\mathrm{g}=30$ and $20 \mu \mathrm{m}$, respectively. Puncture force $\left(F_{\mathrm{p}}\right)$ and displacement $\left(d_{\mathrm{p}}\right)$ were characterized for each array of microneedles. The vertical dashed gray lines divide pre- and post-penetration periods. (Color figure online) 
suggested longer displacement before puncture $\left(d_{\mathrm{p} 1}\right)$, the dull sample might have a deeper dimple before the penetration and then break dura mater and arachnoid at the same time to reach pia mater. On the other hand, the sharp sample might have two steps of penetration: (1) first puncture of dura mater, and (2) another puncture of arachnoid until they reach down to the pia mater. We did not investigate these possibilities further due to the limited resolution of our force gauge.

\subsection{Diverse Cross-Sectional Shapes of Microneedles}

The existing fabrication technologies typically produce microneedles in circular cross sections such as conical or cylindrical shapes [5, 23] and usually do not allow various cross-sectional shapes of final microneedle structures. Since the shape of microneedles is directly related to the stress applied to the skin or brain tissue, the development of various types of microneedles would broaden our understanding of penetrating mechanics as a scientific tool $[2,46$, 48]. For example, a previous study reported, with optimized cross-sectional shapes, can minimize the contact area with the skin, resulting in more reduced puncture resistance and easier insertion [46]. In addition, microneedles in various cross-sectional shapes may be attractive in other applications such as drug delivery [48] and superhydrophobic surfaces [64]. As another scientific tool, microneedle arrays can be used to monitor the physical properties of cell culture platforms [65]. Further applications may include field emission display using Fowler-Nordheim tunneling effect if nanometer scale apex can be realized.

Interestingly, in our proposed approaches, the tip sharpness and the sidewall profile of microneedles were controllable by the photomask design which define areas to be anisotropically etched (Fig. 4e). Thus, we further explored whether cross-sectional shapes of microneedles could be altered by photomask patterns. To implement various crosssectional shapes of microneedle arrays, we tested eight different photomask designs which have distinct microwell opening patterns and their spatial distributions (Fig. 11; quadrilaterally distributed circles, triangularly distributed circles, ellipsoids, triangles, trapezoids, sandglasses, pentagons, and crosses, respectively). Among them, the pattern of quadrilaterally distributed circles was the one used for fabricating microneedles described in previous sections. In each photomask design summarized in Fig. 11, microneedles were formed in the areas highlighted in blue. SEM images in top views and bird's-eye views of Fig. 11 show various microneedle shapes; the final cross-sectional shape of microneedles can be easily controlled by the photomask designs such as pattern shapes and distribution of those shapes. For instance, even with the same size of circles, the final structures of microneedles were substantially distinct depending on their quadrilateral/triangular distribution (Fig. 11a, b). These examples suggest that more intriguing shapes of microneedles can be fabricated by altering photomask designs.

\subsection{Fabrication of Silk Microneedles by Transfer Molding}

To demonstrate a possibility to be used as a drug delivery platform or biodegradable microneedle patches [10, 66], we explored whether our Si microneedle arrays could be expanded to other polymeric materials using a molding process [67]. To promote the releasing process during softlithography, a self-assembled monolayer was formed on microneedles created from the crosses pattern (Fig. 12a). The 10:1 polydimethylsiloxane elastomer (PDMS; Dow Corning Corporation, Midland, MI, USA) was poured on the prepared Si microneedle template (Fig. 12b). After curing, the PDMS replica of the microneedles slowly detached from the Si wafer (Fig. 12c). Next, using the same method with PDMS replica, silk microneedles were fabricated by pouring prepared silk solution (see Methods) on the PDMS mold (Fig. 12d). As shown in Fig. 12e, the initial structure of Si microneedles was successfully transferred to silk microneedles. The transfer molding process can be applicable with other types of biodegradable $[68,69]$ and biocompatible materials [70]. Also, further height modulation of microneedles is expected to enable more effective drug delivery to the epidermis or upper dermis layer by penetrating the stratum corneum without pain $[2,48,71]$.

\section{Conclusions}

In the present study, we have demonstrated the fabrication of Si microneedle arrays that have various cross-sectional shapes and irregular height distributions by using single photolithography and two subsequent DRIE steps. The first 
(a)

Quadrilaterallydistributed circles
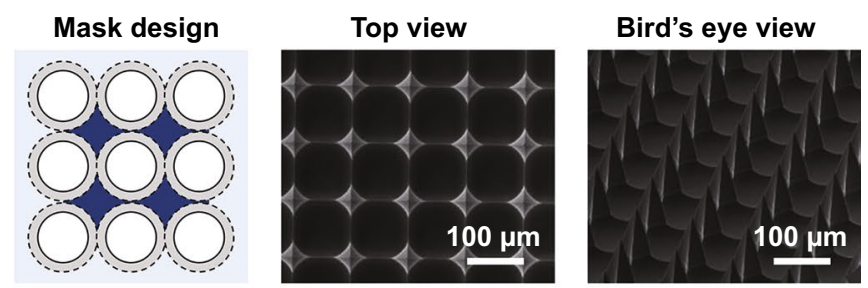

(b)

Triangularlydistributed circles
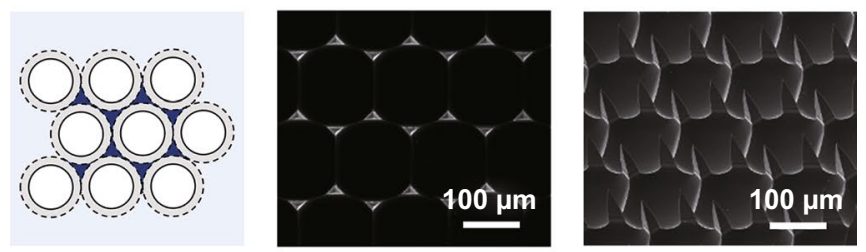

(c)
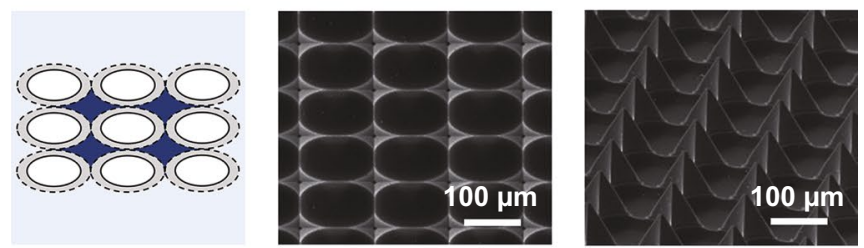

(d)

Triangles
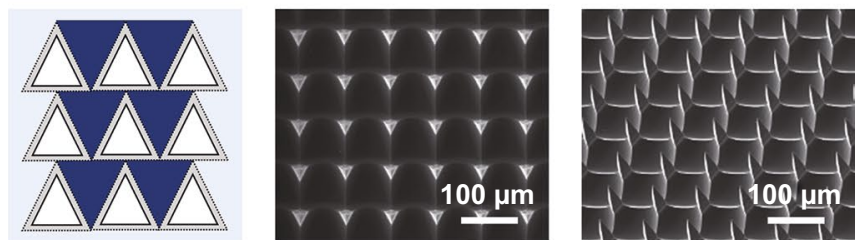

(e)

Trapezoids
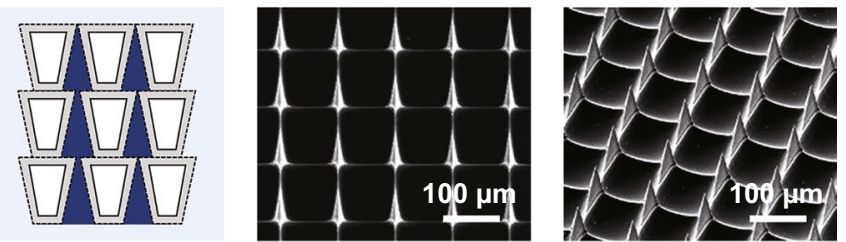

(f)
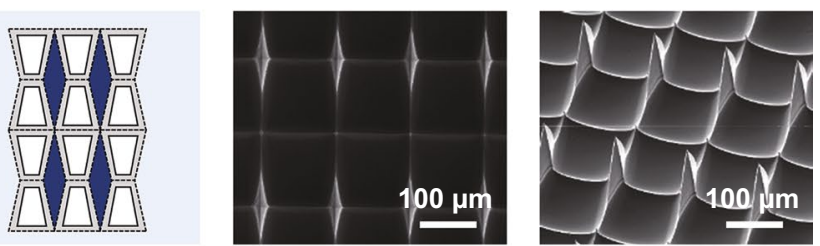

(g)

Sandglasses
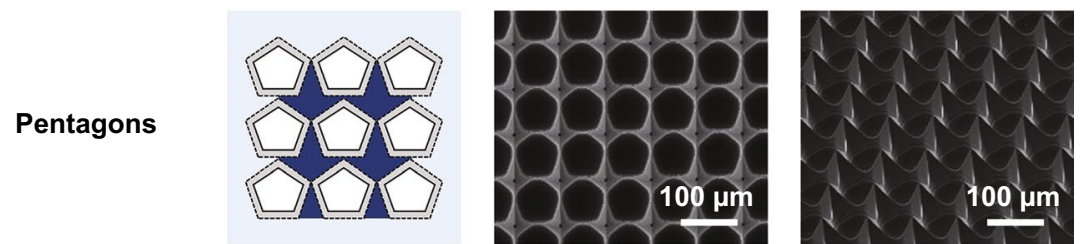

(h)
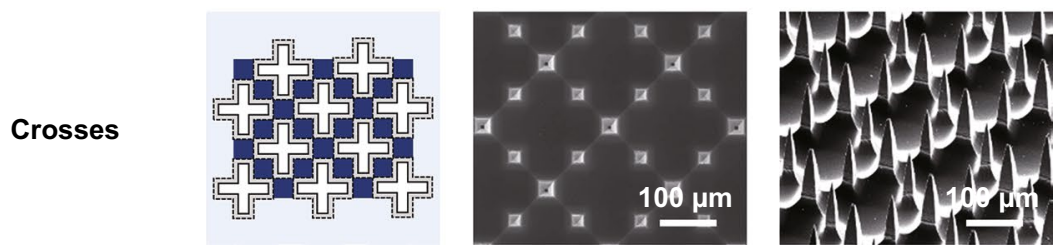

Fig. 11 Fabrication of microneedles that have diverse cross-sectional shapes from various photomask designs. Mask patterns, top and bird's-eye view SEM images are shown for a quadrilaterally distributed circles, $\mathbf{b}$ triangularly distributed circles, $\mathbf{c}$ ellipsoids, $\mathbf{d}$ triangles, e trapezoids, $\mathbf{f}$ sandglasses, $\mathbf{g}$ pentagons, and $\mathbf{h}$ crosses 


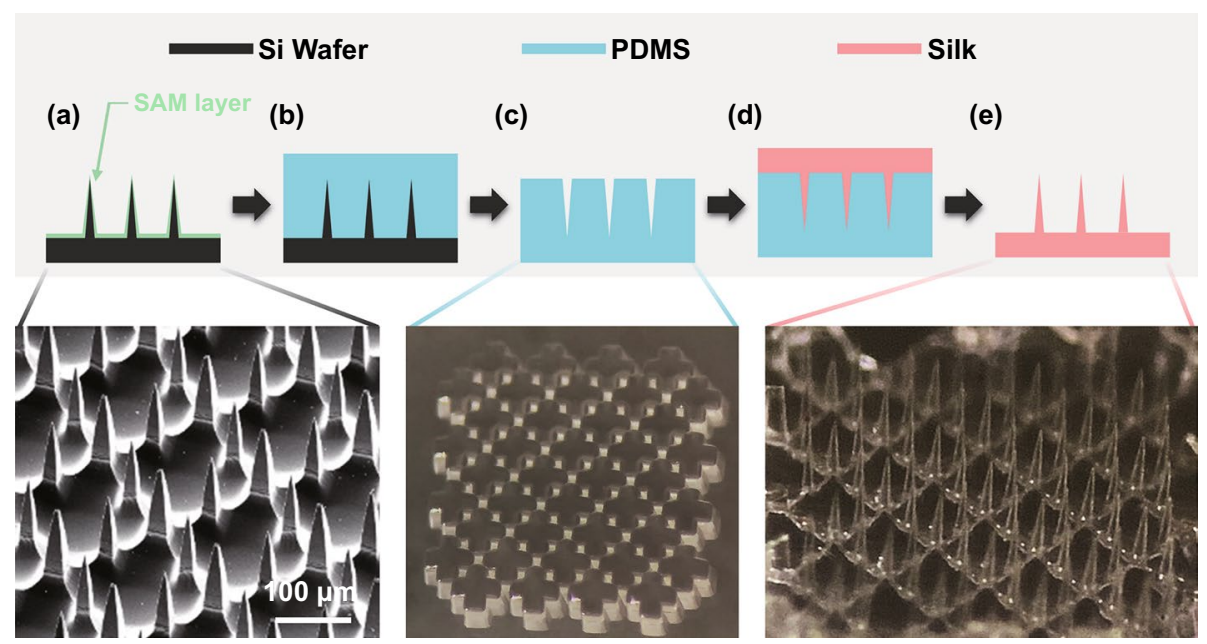

Fig. 12 Fabrication of silk microneedles by transfer molding techniques. a Self-assembled monolayer (SAM) coating on a Si microneedle array. A SEM image of microneedles fabricated from the photomask pattern of crosses is shown at the bottom. b Pouring polydimethylsiloxane (PDMS) elastomer on the Si microneedles. c Detachment of PDMS layer from the Si microneedles. A photograph of the created PDMS mold is shown at the bottom. d Pouring prepared silk solution onto a PDMS replica. e Detachment of the silk microneedles from the PDMS mold. A photograph of the final silk microneedles is shown at the bottom

highly anisotropic DRIE step after photolithography created microwells as an array in a Si wafer. In the 2nd DRIE step, more isotropic etching isolated and sharpened microneedles subsequently. The proposed method which used dry etching processes enabled us to achieve the high density (625 microneedles $\mathrm{mm}^{-2}$ ) of microneedles in a single array, as well as the highest aspect ratio $(\sim 25)$. Both density and aspect ratio may be even more enhanced by optimizing fabrication processes. Also, the final cross-sectional shapes of microneedles were controlled by the shape and distribution of areas to be etching in the 1st DRIE and could be further altered by simply modifying the photomask design. Moreover, the convex/irregular profile of tips of microneedles that have various heights in a given array was fabricated. Those arrays of microneedles in variable lengths can reach various depths of the skin or the brain to promote more effective with adequate penetration depths. It is expected to be more useful if those microneedles are monolithically packaged with integrated circuits fabricated on a solid Si substrate to access highly curved tissues. By insertion test of microneedles using agarose gels and a mouse brain, the required insertion force was characterized. The fabricated microneedle array required only $172 \mu \mathrm{N}$ per one microneedle to penetrate the mouse brain. Taken all together, our new fabrication method can facilitate the manufacture of microneedle arrays for various applications including drug delivery, neurophysiological/neuroprosthetic researches, and so on. The use of mature semiconductor fabrication processes is expected to enable the creations of microneedles in a large area.

Acknowledgements Authors thank all staff members of KIST Micro/Nano Fabrication Center for technical supports. This work was supported by KIST (Korea Institute of Science and Technology) institutional grants (2E30965, and 2V07360), the National R\&D Program through the National Research Foundation of Korea (NRF) funded by the Ministry of Science and ICT (Nos. 2020R1C1C1006065, 2021M3F3A2A01037366). This work was also supported by the Korea Medical Device Development Fund grant funded by the Korea government (the Ministry of Science and ICT, the Ministry of Trade, Industry and Energy, the Ministry of Health \& Welfare, the Ministry of Food and Drug Safety) (Project Number: 9991006818, KMDF_PR_20200901_0145-2021).

Funding Open access funding provided by Shanghai Jiao Tong University.

Open Access This article is licensed under a Creative Commons Attribution 4.0 International License, which permits use, sharing, adaptation, distribution and reproduction in any medium or format, as long as you give appropriate credit to the original author(s) and the source, provide a link to the Creative Commons licence, and indicate if changes were made. The images or other third party material in this article are included in the article's Creative Commons licence, unless indicated otherwise in a credit line to the material. If material is not included in the article's Creative Commons licence and your intended use is not permitted by statutory regulation or exceeds the permitted use, you will need to obtain permission directly from the copyright holder. To view a copy of this licence, visit http://creativecommons.org/licenses/by/4.0/. 


\section{References}

1. P. Van Damme, F. Oosterhuis-Kafeja, M. Van der Wielen, Y. Almagor, O. Sharon et al., Safety and efficacy of a novel microneedle device for dose sparing intradermal influenza vaccination in healthy adults. Vaccine 27(3), 454-459 (2009). https://doi.org/10.1016/j.vaccine.2008.10.077

2. T. Waghule, G. Singhvi, S.K. Dubey, M.M. Pandey, G. Gupta et al., Microneedles: a smart approach and increasing potential for transdermal drug delivery system. Biomed. Pharmacotherapy 109, 1249-1258 (2019). https://doi.org/10.1016/j.biopha. 2018.10.078

3. Y. Hasegawa, Y. Yasuda, K. Taniguchi, M. Shikida, Fabrication method with high-density, high-height microneedle using microindentation method for drug delivery system. Microsys. Technol. 26, 765-773 (2020). https://doi.org/10. 1007/s00542-019-04571-4

4. Y. Lee, S.R. Dugansani, S.H. Jeon, S.H. Hwang, J.-H. Kim et al., Drug-delivery system based on salmon DNA nano- and micro-scale structures. Sci. Rep. 7, 9724 (2017). https://doi. org/10.1038/s41598-017-09904-9

5. M. Bok, Y. Lee, D. Park, S. Shin, Z.-J. Zhao et al., Microneedles integrated with a triboelectric nanogenerator: an electrically active drug delivery system. Nanoscale 10, 13502-13510 (2018). https://doi.org/10.1039/c8nr02192a

6. W.-G. Bae, H. Ko, J.-Y. So, H. Yi, C.-H. Lee et al., Snake fang-inspired stamping patch for transdermal delivery of liquid formulations. Sci. Transl. Med. 11(503), eaaw3329 (2019). https://doi.org/10.1126/scitranslmed.aaw3329

7. Y. Gao, M. Hou, R. Yang, L. Zhang, Z. Xu et al., PEGDA/ PVP microneedles with tailorable matrix constitutions for controllable transdermal drug delivery. Macromol. Mater. Eng. 303(12), 1800233 (2018). https://doi.org/10.1002/mame. 201800233

8. C.J.W. Bolton, O. Howells, G.J. Blayney, P.F. Eng, J.C. Birchall et al., Hollow silicon microneedle fabrication using advanced plasma etch technologies for applications in transdermal drug delivery. Lab Chip 20(15), 2788 (2020). https:// doi.org/10.1039/d01c00567c

9. E. Kim, G. Erdos, S. Huang, T.W. Kenniston, S.C. Balmert et al., Microneedle array delivered recombinant coronavirus vaccines: Immunogenicity and rapid translational development. EBioMedicine 55, 102743 (2020). https://doi.org/10. 1016/j.ebiom.2020.102743

10. M.D. Shin, S. Shukla, Y.H. Chung, V. Beiss, S.K. Chan et al., COVID-19 vaccine development and a potential nanomaterial path forward. Nat. Nanotechnol. 15, 646-655 (2020). https:// doi.org/10.1038/s41565-020-0737-y

11. K.J. Lee, S.H. Park, J.Y. Lee, H.C. Joo, E.H. Jang et al., Perivascular biodegradable microneedle cuff for reduction of neointima formation after vascular injury. J. Con. Release 192(28), 174-181 (2014). https://doi.org/10.1016/j.jconrel. 2014.07.007

12. J. Yu, Y. Zhang, Y. Ye, R. DiSanto, W. Sun et al., Microneedle-array patches loaded with hypoxia-sensitive vesicles provide fast glucose-responsive insulin delivery. PNAS
112(27), 8260-8265 (2015). https://doi.org/10.1073/pnas. 1505405112

13. K. Lee, H.B. Song, W. Cho, J.H. Kim, J.H. Kim et al., Intracorneal injection of a detachable hybrid microneedle for sustained drug delivery. Acta Biomater. 80(15), 48-57 (2018). https://doi.org/10.1016/j.actbio.2018.09.039

14. J. Tang, J. Wang, K. Huang, Y. Ye, T. Su et al., Cardiac cellintegrated microneedle patch for treating myocardial infarction. Sci. Adv. 4(11), eaat9365 (2018). https://doi.org/10.1126/ sciadv.aat9365

15. Y. Cao, E. Lim, M. Xu, J.-K. Weng, B. Marelli, Precision delivery of multiscale payloads to tissue-specific targets in plants. Adv. Sci. 7(13), 1903551 (2020). https://doi.org/10. 1002/advs.201903551

16. R. Paul, A.C. Saville, J.C. Hansel, Y. Ye, C. Ball et al., Extraction of plant DNA by microneedle patch for rapid detection of plant diseases. ACS Nano 13(6), 6540-6549 (2019). https:// doi.org/10.1021/acsnano.9b00193

17. A. Vázquez-Guardado, Y. Yang, A.J. Bandodkar, J.A. Rogers, Recent advances in neurotechnologies with broad potential for neuroscience research. Nat. Neurosci. 23, 1522-1536 (2020). https://doi.org/10.1038/s41593-020-00739-8

18. G. Charvet, L. Rousseau, O. Billoint, S. Gharbi, J.-P. Rostaing et al., BioMEA ${ }^{\mathrm{TM}}$ : a versatile high-density $3 \mathrm{D}$ microelectrode array system using integrated electronics. Biosens. Bioelectron. 25(8), 1889-1896 (2010). https://doi.org/10.1016/j.bios. 2010.01.001

19. R.A. Normann, E. Fernandez, Clinical applications of penetrating neural interfaces and Utah electrode array technologies. J. Neural Eng. 13(6), 061003 (2016). https://doi.org/10. 1088/1741-2560/13/6/061003

20. R. Wang, X. Jiang, W. Wang, Z. Li, A microneedle electrode array on flexible substrate for long-term EEG monitoring. Sens. Actuators B Chem. 244, 750-758 (2017). https://doi. org/10.1016/j.snb.2017.01.052

21. X. Chen, F. Wang, E. Fernandez, P.R. Roelfsema, Shape perception via a high-channel-count neuroprosthesis in monkey visual cortex. Science 370(6521), 1191-1196 (2020). https:// doi.org/10.1126/science.abd7435

22. Z. Xiang, J. Liu, C. Lee, A flexible three-dimensional electrode mesh: an enabling technology for wireless brain-computer interface prostheses. Microsyst. Nanoeng. 2, 16012 (2016). https://doi.org/10.1038/micronano.2016.12

23. H.A.C. Wark, R. Sharma, K.S. Mathews, E. Fernandez, J. Yoo et al., A new high-density ( 25 electrodes $/ \mathrm{mm}^{2}$ ) penetrating microelectrode array for recording and stimulating sub-millimeter neuroanatomical structures. J. Neural Eng. 10(4), 045003 (2013). https://doi.org/10.1088/1741-2560/10/4/045003

24. S. Micera, J. Carpaneto, S. Raspopovic, Control of hand prostheses using peripheral information. IEEE Rev. Biomed. Eng. 3, 48-68 (2010). https://doi.org/10.1109/RBME.2010. 2085429

25. S.Y. Yang, E.D. O'Cearbhaill, G.C. Sisk, K.M. Park, W.K. Cho et al., A bio-inspired swellable microneedle adhesive for mechanical interlocking with tissue. Nat. Comm. 4, 1702 (2013). https://doi.org/10.1038/ncomms 2715 
26. M. Chiaranairungroj, A. Pimpin, W. Srituravanich, Fabrication of high-density microneedle masters towards the commercialisation of dissolving microneedles. Micro Nano Lett. 13(3), 284-288 (2018). https://doi.org/10.1049/mnl.2017. 0596

27. P. Makvandi, M. Kirkby, A.R.J. Hutton, M. Shabani, C.K.Y. Yiu et al., Engineering microneedle patches for improved penetration: Analysis, skin models and factors affecting needle insertion. Nano-Micro Lett. 13, 93 (2021). https://doi.org/10. 1007/s40820-021-00611-9

28. R.T. Narayanan, D. Udvary, M. Oberlaender, Cell type-specific structural organization of the six layers in rat barrel cortex. Front. Neuroanat. 11, 91 (2017). https://doi.org/10.3389/fnana. 2017.00091

29. S. Raspopovic, M. Capogrosso, J. Badia, X. Navarro, S. Micera, Experimental validation of a hybrid computational model for selective stimulation using transverse intrafascicular multichannel electrodes. IEEE Trans. Neural Syst. Rehabil. Eng. 20(3), 395-404 (2012). https://doi.org/10.1109/TNSRE. 2012.2189021

30. R. Bhandari, S. Negi, L. Rieth, R.A. Normann, F. Solzbacher, A novel method of fabricating convoluted shaped electrode arrays for neural and retinal prostheses. Sens. Actuators A Phys. 145-146, 123-130 (2008). https://doi.org/10.1016/j.sna. 2007.10.072

31. K.-W. Lee, Y. Watanabe, C. Kigure, T. Fukushima, M. Koyanagi et al., Pillar-shaped stimulus electrode array for highefficiency stimulation of fully implantable epiretinal prosthesis. J. Micromech. Microeng. 22(10), 105015 (2012). https:// doi.org/10.1088/0960-1317/22/10/105015

32. H. Yu, W. Xiong, H. Zhang, W. Wang, Z. Li, A parylene self-locking cuff electrode for peripheral nerve stimulation and recording. J. Microelectromech. Syst. 23(5), 1025-1035 (2014). https://doi.org/10.1109/JMEMS.2014.2333733

33. M.A. Lebedev, M.A.L. Nicolelis, Brain-machine interfaces: past, present and future. Trends Neurosci. 29(9), 536-546 (2006). https://doi.org/10.1016/j.tins.2006.07.004

34. P.J. Rousche, R.A. Normann, A method for pneumatically inserting an array of penetrating electrodes into cortical tissue. Ann. Biomed. Eng. 20, 413-422 (1992). https://doi.org/ 10.1007/BF02368133

35. R.C. Kelly, M.A. Smith, J.M. Samonds, A. Kohn, A.B. Bonds et al., Comparison of recordings from microelectrode arrays and single electrodes in the visual cortex. J. Neurosci. 27(2), 261-264 (2007). https://doi.org/10.1523/JNEUROSCI.490606.2007

36. A. Obaid, Y.-W. Wu, M. Hanna, W. Nix, J. Ding et al., Ultrasensitive measurement of brain penetration with microscale probes for brain machine interface considerations. bioRxiv (2018). https://doi.org/10.1101/454520

37. P. Khanna, K. Luongo, J.A. Strom, S. Bhansali, Sharpening of hollow silicon microneedles to reduce skin penetration force. J. Micromech. Microeng. 20(4), 045011 (2010). https://doi. org/10.1088/0960-1317/20/4/045011

38. A. Boonma, R.J. Narayan, Y.-S. Lee, Analytical modeling and evaluation of microneedles apparatus with deformable soft tissues for biomedical applications. Comput. Aided Des. Appl. 10(1), 139-157 (2013). https://doi.org/10.3722/cadaps.2013. 139-157

39. B. Vandekerckhove, J. Missinne, K. Vonck, P. Bauwens, R. Verplancke et al., Technological challenges in the development of optogenetic closed-loop therapy approaches in epilepsy and related network disorders of the brain. Micromachines 12(1), 38 (2021). https://doi.org/10.3390/mi12010038

40. D.J. Edell, V.V. Toi, V.M. McNeil, L.D. Clark, Factors influencing the biocompatibility of insertable silicon microshafts in cerebral cortex. IEEE Trans. Biomed. Eng. 39(6), 635-643 (1992). https://doi.org/10.1109/10.141202

41. C.S. Bjornsson, S.J. Oh, Y.A. Al-Kofahi, Y.J. Lim, K.L. Smith et al., Effects of insertion conditions on tissue strain and vascular damage during neuroprosthetic device insertion. J. Neural Eng. 3(3), 196-207 (2006). https://doi.org/10.1088/ $1741-2560 / 3 / 3 / 002$

42. A.A. Sharp, A.M. Ortega, D. Restrepo, D. Curran-Everett, K. Gall, In vivo penetration mechanics and mechanical properties of mouse brain tissue at micrometer scales. IEEE Trans. Biomed. Eng. 56(1), 45-53 (2009). https://doi.org/10.1109/ TBME.2008.2003261

43. Y. Li, H. Zhang, R. Yang, Y. Laffitte, U. Schmill et al., Fabrication of sharp silicon hollow microneedles by deepreactive ion etching towards minimally invasive diagnostics. Microsys. Nanoeng. 5, 41 (2017). https://doi.org/10.1038/ s41378-019-0077-y

44. R. Khilwani, P.J. Gilgunn, T.D.Y. Kozai, X.C. Ong, E. Korkmaz et al., Ultra-miniature ultra-compliant neural probes with dissolvable delivery needles: design, fabrication and characterization. Biomed. Microdev. 18, 97 (2016). https:// doi.org/10.1007/s10544-016-0125-4

45. J.W. Salatino, K.A. Ludwig, T.D.Y. Kozai, E.K. Purcell, Glial responses to implanted electrodes in the brain. Nat. Biomed. Eng. 1, 862-877 (2017). https://doi.org/10.1038/ s41551-017-0154-1

46. S. Aoyagi, K. Okuda, T. Takahashi, M. Suzuki, Effect of microneedle cross-sectional shape on puncture resistanceinvestigation of polygonal and star-shaped cross sections -. J. Robotics Mechatronics 32(2), 371-381 (2020). https://doi. org/10.20965/jrm.2020.p0371

47. R. Bhandari, S. Negi, L. Rieth, F. Solzbacher, A wafer-scale etching technique for high aspect ratio implantable MEMS structures. Sens. Actuators A Phys. 162(1), 130-136 (2010). https://doi.org/10.1016/j.sna.2010.06.011

48. N. Roxhed, T.C. Gasser, P. Griss, G.A. Holzapfel, G. Stemme, Penetration-enhanced ultrasharp microneedles and prediction on skin interaction for efficient transdermal drug delivery. J. Microelectromech. Syst. 16(6), 1429-1440 (2007). https://doi. org/10.1109/JMEMS.2007.907461

49. K. Lee, H.C. Lee, D.-S. Lee, H. Jung, Drawing lithography: three-dimensional fabrication of an ultrahigh-aspect-ratio microneedle. Adv. Mater. 22(4), 483-486 (2010). https://doi. org/10.1002/adma.200902418

50. J. Lee, S.H. Park, I.H. Seo, K.J. Lee, W. Ryu, Rapid and repeatable fabrication of high $\mathrm{A} / \mathrm{R}$ silk fibroin microneedles 
using thermally-drawn micromolds. Eur. J. Pharm. Biopharm. 94, 11-19 (2015). https://doi.org/10.1016/j.ejpb.2015.04.024

51. H. Wang, Z. Xiang, C.-F. Hu, G. Pastorin, W. Fang et al., Microneedle array integrated with CNT nanofilters for controlled and selective drug delivery. J. Microelectromech. Syst. 23(5), 1036-1044 (2014). https://doi.org/10.1109/JMEMS. 2014.2339212

52. N. Roxhed, P. Griss, G. Stemme, A method for tapered deep reactive ion etching using a modified Bosch process. J. Micromech. Microeng. 17(5), 1087-1092 (2007). https://doi.org/10. 1088/0960-1317/17/5/031

53. R. Nagarajan, L. Ebin, L. Dayong, S.C. Seng, K. Prasad, Development of a novel deep silicon tapered via etch process for through-silicon interconnection in 3-D integrated systems. Proc. 56th Electronic Components and Tech. Conf. (San Diego, CA, USA) 383-387 (2006). http://doi.org/https:// doi.org/10.1109/ECTC.2006.1645674

54. R.F. Figueroa, S. Spiesshoefer, S.L. Burkett, L. Schaper, Control of sidewall slope in silicon vias using $\mathrm{SF}_{6} / \mathrm{O}_{2}$ plasma etching in a conventional reactive ion etching tool. J. Vac. Sci. Technol. B 23(5), 2226-2231 (2005). https://doi.org/10. 1116/1.2041654

55. Z. Xiang, J. Liu, C. Lee, A flexible three-dimensional electrode mesh: An enabling technology for wireless brain-computer interface prostheses. Microsyst. Nanoeng. 2, 16012 (2016). https://doi.org/10.1038/micronano.2016.12

56. R. Li, Y. Lamy, W.F.A. Besling, F. Roozeboom, P.M. Sarro, Continuous deep reactive ion etching of tapered via holes for three-dimensional integration. J. Micromech. Microeng. 18, 125023 (2008). https://doi.org/10.1088/0960-1317/18/12/ 125023

57. S. Seok, B. Lee, J. Kim, H. Kim, K. Chun, A new compensation method for the footing effect in MEMS fabrication. J. Micromech. Microeng. 15, 1791-1796 (2005). https://doi.org/ 10.1088/0960-1317/15/10/001

58. E.M. Maynard, Visual prostheses. Annu. Rev. Biomed. Eng. 3, 145-168 (2001). https://doi.org/10.1146/annurev.bioeng.3.1. 145

59. D. Boinagrov, S. Pangratz-Fuehrer, G. Goetz, D. Palanker, Selectivity of direct and network-mediated stimulation of the retinal ganglion cells with epi-, sub- and intraretinal electrodes. J. Neural Eng. 11(2), 026008 (2014). https://doi.org/ $10.1088 / 1741-2560 / 11 / 2 / 026008$

60. M. Im, S.I. Fried, Indirect activation elicits strong correlations between light and electrical responses in $\mathrm{ON}$ but not $\mathrm{OFF}$ retinal ganglion cells. J. Physiol. 593(16), 3577-3596 (2015). https://doi.org/10.1113/JP270606

61. M. Im, S.I. Fried, Temporal properties of network-mediated responses to repetitive stimuli are dependent upon retinal ganglion cell type. J. Neural Eng. 13(2), 025002 (2016). https://doi.org/10.1088/1741-2560/13/2/025002

62. Z. Chen, Y. Lin, W. Lee, L. Ren, B. Liu et al., Additive manufacturing of honeybee-inspired microneedle for easy skin insertion and difficult removal. ACS Appl. Mater. Interfaces 10(35), 29338-29346 (2018). https://doi.org/10.1021/acsami. 8 b09563

63. Z. Fekete, A. Németh, G. Márton, I. Ulbert, A. Pongrácz, Experimental study on the mechanical interaction between silicon neural microprobes and rat dura mater during insertion. J. Mater. Sci. Mater. Med. 26, 70 (2015). https://doi.org/ 10.1007/s10856-015-5401-y

64. M. Im, H. Im, J.-H. Lee, J.-B. Yoon, Y.-K. Choi, A robust superhydrophobic and superoleophobic surface with invertrapezoidal microstructures on a large transparent flexible substrate. Soft Matter 6(7), 1401-1404 (2010). https://doi. org/10.1039/B925970H

65. H. Seong, S.G. Higgins, J. Penders, J.P.K. Armstrong, S.W. Crowder et al., Size-tunable nanoneedle arrays for influencing stem cell morphology, gene expression, and nuclear membrane curvature. ACS Nano 14(5), 5371-5381 (2020). https://doi. org/10.1021/acsnano.9b08689

66. J.-H. Park, M.G. Allen, M.R. Prausnitz, Biodegradable polymer microneedles: Fabrication, mechanics and transdermal drug delivery. J. Control. Release 104(1), 51-66 (2005). https://doi.org/10.1016/j.jconrel.2005.02.002

67. M. Mahmood, S. Kwon, H. Kim, Y.-S. Kim, P. Siriaraya et al., Wireless soft scalp electronics and virtual reality system for motor imagery-based brain-machine interfaces. Adv. Sci. 8(19), 2101129 (2021). https://doi.org/10.1002/advs.20210 1129

68. S. Aoyagi, H. Izumi, M. Fukuda, Biodegradable polymer needle with various tip angles and consideration on insertion mechanism of mosquito's proboscis. Sens. Actuators A Phys. 143(1), 20-28 (2008). https://doi.org/10.1016/j.sna.2007.06. 007

69. F. Ceyssens, M. Welkenhuysen, R. Puers, Anisotropic etching in $\left(\begin{array}{lll}3 & 1 & 1\end{array}\right) \mathrm{Si}$ to fabricate sharp resorbable polymer microneedles carrying neural electrode arrays. J. Micromech. Microeng. 29(2), 027001 (2019). https://doi.org/10.1088/1361-6439/ AAF43A

70. X. Hong, Z. Wu, L. Chen, F. Wu, L. Wei et al., Hydrogel microneedle arrays for transdermal drug delivery. Nano-Micro Lett. 6, 191-199 (2014). https://doi.org/10.5101/nml140021r

71. T. Someya, M. Amagai, Toward a new generation of smart skins. Nat. Biotechnol. 37, 382-388 (2019). https://doi.org/ 10.1038/s41587-019-0079-1 